Y. Matsushima

Nagoya Math. J.

Vol. 61 (1976), 161-195

\title{
HEISENBERG GROUPS AND HOLOMORPHIC VECTOR BUNDLES OVER A COMPLEX TORUS
}

\author{
YOZO MATSUSHIMA
}

Let $V$ be a complex vector space of dimension $n, L$ a lattice of $V$ and $E=V / L$ a complex torus. Let $H$ be a Hermitian form on $V$. We introduce a multiplication in $L \times C^{*}$ by

$$
(\alpha, a)(\beta, b)=(\alpha+\beta,(\exp \pi H(\alpha, \beta)) a b),
$$

where $\alpha, \beta \in L$ and $a, b \in C^{*}$. Then $L \times C^{*}$ becomes a complex Lie group $G_{H}(L)$ whose identity component is $C^{*}$. We call $G_{H}(L)$ the Heisenberg group associated with a Hermitian form $H$ and a lattice $L$ in $V$. In general $G_{H}(L)$ is non-abelian. The group $G_{H}(L)$ acts on the complex manifold $V \times C^{*}$ from the right by the rule

$$
(u, a)(\beta, b)=(u+\beta,(\exp \pi H(u, \beta)) a b),
$$

where $u \in V, \beta \in L$ and $a, b \in C^{*}$. The action of $G_{H}(L)$ is holomorphic and free and we can identify the quotient space with the complex torus $E$. Thus $V \times C^{*}$ is a principal fibre bundle over $E$ with structure group $G_{H}(L)$. If we vary the Hermitian form $H$, we obtain infinitely many principal holomorphic bundle structures over $E$ in this manner.

The purpose of this article is to study the class of holomorphic vector bundles over $E$ associated with holomorphic representations of the Heisenberg groups $G_{H}(L)$.

If a representation of $G_{H}(L)$ is trivial on $\{0\} \times C^{*}$, the representation is nothing but a representation of the lattice $L$. The vector bundles over $E$ associated with representations of $L$ have been studied in our previous paper [2]. In this paper we shall show first that every holomorphic line bundle over $E$ is always associated with a holomorphic representation of degree 1 of the Heisenberg group $G_{H}(L)$ for a suitable Hermitian form $H$. This result is nothing but an interpretation of the

Received August 4, 1975. 
"normalized" theta factor associated with a line bundle. Then we show that if the Heisenberg group $G_{H}(L)$ admits an irreducible holomorphic representation which is not trivial on $\{0\} \times C^{*}$, the imaginary part $A$ of $H$, which is an alternating form on $V$, is rational valued on $L$. If $A$ is integral valued on $L$, the group $G_{H}(L)$ is abelian and so holomorphic irreducible representations are of degree 1 and the associated bundles are line bundles. However if $A$ is not integral valued but rational valued on $L$, there are holomorphic irreducible representations of $G_{H}(L)$ of degree greater than one. In the sections 5 and 6 we classify the holomorphic irreducible representations of the Heisenberg group $G_{H}(L)$ assuming that the imaginary part $A$ of $H$ is rational valued on $L$. To achieve this classification we introduce another multiplication on $L \times C^{*}$ by

$$
(\alpha, a)(\beta, b)=(\alpha+\beta,(\exp \pi i A(\alpha, \beta)) a b) .
$$

Then $L \times C^{*}$ becomes also a complex Lie group $G_{A}(L)$ with respect to this new multiplication. We call also $G_{A}(L)$ the Heisenberg group associated with $A$ and $L$. It is easy to show that $G_{H}(L)$ and $G_{A}(L)$ are isomorphic (see $\S 2$ ) and consequently there is a one-to-one correspondence between holomorphic representations of $G_{H}(L)$ and those of $G_{A}(L)$. We classify holomorphic irreducible representations of $G_{A}(L)$ in the sections 5 and 6. Among the holomorphic irreducible representations of the Heisenberg group $G_{A}(L)$ there is a distinguished representation $D_{A}$ which we call the Schrödinger representation. The representation $D_{H}$ of $G_{H}(L)$ which corresponds to $D_{A}$ is also called the Schrödinger representation of $G_{H}(L)$. In the section 7 we shall show that a holomorphic vector bundle over $E$ associated with a holomorphic irreducible representation of $G_{H}(L)$ is isomorphic to a holomorphic vector bundle associated with a representation of $G_{k_{H}}(L)$ of the form $\sigma \otimes D_{k_{H}}$, where $\sigma$ is a 1-dimensional representation of the lattice $L, D_{k H}$ is the Schrödinger representation of $G_{k H}(L)$ and $k$ is a suitable integer.

In the section 8 we study some properties of the vector bundle $F$ associated with the Schrödinger representation $D_{H}$. The vector bundle $F$ is simple and hence indecomposable. We study the mechanism to construct the vector valued theta functions associated with $F$. It will be shown for example, that if the Hermitian form $H$ is positive $(\geqq 0)$, then the number of linearly independent theta functions is given by 


$$
\prod_{i=1}^{\ell} e_{i}\left(e_{i}, d\right)^{-1}
$$

whereas the rank of the vector bundle $F$ is

$$
d^{\ell} \prod_{i=1}^{\ell}\left(e_{i}, d\right)^{-1},
$$

where $d$ is the smallest positive integer such that $d A$ is integral valued on $L$ and $e_{1}, \cdots, e_{\ell}$ are non-zero elementary divisors of the integral alternating form $d A$ on $L$.

In the final section we study the properties of tensor products of vector bundles associated with indecomposable holomorphic representations of $G_{H}(L)$ for variable $H$ and we compute Chern classes. We shall see that, if $F_{1}$ and $F_{2}$ are vector bundles associated with the Schrödinger representations of $G_{H_{1}}(L)$ and $G_{H_{2}}(L)$, then we have the splitting $F_{1} \otimes F_{2}$ $\cong F_{\sigma_{1} \otimes D_{H}} \oplus \cdots \oplus F_{\sigma_{s} \otimes D_{H}}$, where $H=H_{1}+H_{2}, D_{H}$ is the Schrödinger representation of $G_{H}(L), \sigma_{1}, \cdots, \sigma_{s}$ are 1-dimensional representations of $L$ and $F_{\sigma_{i} \otimes D_{H}}$ denotes the vector bundle associated with the representation $\sigma_{i} \otimes D_{H}$ of $G_{H}(L)$.

The group $G_{A}(V)$ appeared already in a paper of Murakami [8] in a similar context as ours and this article is also closely related with the works of Morikawa [3] and Oda [5]. The author wishes to thank J. Hano for his useful comments. J. Hano also proved recently that the class of vector bundles studied by Morikawa and Oda is identical with the one associated with irreducible representations of $G_{H}(L)$.

\section{§ 1. The nilpotent Lie group $G_{B}$.}

Let $V$ be a finite dimensional vector space over $R$ and $B$ a complex valued bilinear form on $V$. We define a multiplication in the product $V \times C^{*}$ by

$$
(u, a)(v, b)=\left(u, v, \varepsilon\left\{\frac{1}{2} B(u, v)\right\} a b\right)
$$

where $u, v \in V$ and $a, b \in C^{*}$ and

$$
\varepsilon(z)=\exp 2 \pi i z
$$

for all $z \in C$.

With respect to the multiplication (1.1) $V \times C^{*}$ forms a Lie group 
which we shall denote by $G_{B}$. The element $(0,1)$ is the identity element and the inverse of $(u, a)$ is given by the formula

$$
(u, a)^{-1}=\left(-u, \varepsilon\left\{\frac{1}{2} B(u, u)\right\} a^{-1}\right) .
$$

Let $B=S+A$, where $S(u, v)=\frac{1}{2}\{B(u, v)+B(v, u)\}$ is a symmetric bilinear form and $A(u, v)=\frac{1}{2}\{B(u, v)-B(v, u)\}$ is an alternating bilinear form. From (1.1) we get the following commutation rule:

$$
(u, a)(v, b)=(v, b)(u, a)(0, \varepsilon\{A(u, v)\}) .
$$

The subset $\{0\} \times C^{*}$ form a closed normal subgroup contained in the center of $G_{B}$ and the quotient of $G_{B}$ by $\{0\} \times C^{*}$ is an abelian Lie group isomorphic to $V$. Hence $G_{B}$ is a connected nilpotent Lie group and $G_{B}$ is not abelian unless $A=0$.

We can also define a nilpotent Lie group $G_{A}$ by introducing another multiplication in $V \times C^{*}$ by

$$
(u, a)(v, b)=\left(u+v, \varepsilon\left\{\frac{1}{2} A(u, v)\right\} a b\right) .
$$

We can prove easily the following lemma.

LEMMA 1.1. The map $\varphi$ from $G_{B}$ onto $G_{A}$ given by

$$
\varphi(u, a)=\left(u, \varepsilon\left\{-\frac{1}{4} B(u, u)\right\} a\right)
$$

is an isomorphism of $G_{B}$ onto $G_{A}$.

\section{§ 2. The Heisenberg group $G_{H}(L)$.}

We now assume that $V$ is a complex vector space of complex dimension $n$ and let $H$ be a Hermitian form on $V$. Let

$$
H(u, v)=S(u, v)+i A(u, v),
$$

for $u, v \in V$, where $S(u, v)$ and $A(u, v)$ are the real part and the imaginary part of $H(u, v)$. Then $S$ is symmetric and $A$ is alternating, both are $\boldsymbol{R}$-bilinear on $V$. Let 


$$
B=\frac{1}{i} H
$$

and we define the nilpotent Lie group $G_{B}$ as in $\S 1$ which we shall denote by $G_{H}$. Thus the multiplication in the group $G_{H}$ is defined by

$$
(u, a)(v, b)=\left(u, v, \varepsilon\left\{\frac{1}{2 i} H(u, v)\right\} a b\right),
$$

where $u, v \in V$ and $a, b \in C^{*}$.

Since $H$ is Hermitian, the multiplication in $G_{H}$ is holomorphic in the variable $u, a$ and $b$ and anti-holomorphic in the variable $v$. Hence $G_{H}$ is not a complex Lie group.

The alternating part of $B=\frac{1}{i} H$ is equal to the imaginary part $A$ of $H$ and by Lemma 1.1. the map $\varphi: G_{H} \rightarrow G_{A}$ defined by

$$
\varphi(u, a)=\left(u, \varepsilon\left\{-\frac{1}{4 i} H(u, u)\right\} a\right)
$$

is an isomorphism of $G_{H}$ onto $G_{A}$.

Let $L$ be a lattice of $V$ and we define

$$
G_{H}(L)=L \times C^{*} .
$$

Then $G_{H}(L)$ is a closed subgroup of $G_{H}$ and $G_{H}(L)$ is a complex Lie group with the identity component isomorphic to $C^{*}$.

Analogously

$$
G_{A}(L)=L \times C^{*}
$$

is also a subgroup of $G_{A}$ and $G_{A}(L)$ is also a complex Lie group. Moreover the isomorphism $\varphi$ of $G_{H}$ onto $G_{A}$ defined by (2.2) maps $G_{H}(L)$ onto $G_{A}(L)$ and induces an isomorphism of complex Lie group.

We call $G_{H}(L)$ (resp. $G_{A}(L)$ ) the Heisenberg group associated with a Hermitian form $H$ and a lattice $L$ (resp. $A$ and $L$ ).

\section{§ 3. Principal bundle structures over a complex torus associated with Heisenberg groups.}

Since $G_{H}(L)$ is a subgroup of $G_{H}$, it acts on $G_{H}=V \times C^{*}$ freely by right multiplication 


$$
(u, a)(\beta, b)=\left(u+\beta, \varepsilon\left\{\frac{1}{2 i} H(u, \beta)\right\} a b\right),
$$

where $u \in V, \beta \in L$ and $a, b \in C^{*}$. This is a holomorphic and free action of the complex Lie group $G_{H}(L)$ on the complex manifold $V \times C^{*}$ and we can identify the quotient space canonically with the complex torus

$$
E=V / L \text {. }
$$

Thus $L \times C^{*}$ is a holomorphic principal fibre bundle over the complex torus $E$ with the structure group $G_{H}(L)$.

A $G_{H}$-theta factor $\tilde{J}$ of rank $m$ is a holomorphic map

$$
\tilde{J}: G_{H}(L) \times V \times C^{*} \rightarrow G L_{m}(C)
$$

such that

$$
\tilde{J}\left(\gamma \gamma^{\prime}, g\right)=\tilde{J}\left(\gamma, T_{r^{\prime}} g\right) \tilde{J}\left(\gamma^{\prime}, g\right)
$$

for $\gamma, \gamma^{\prime} \in G_{H}(L)$ and $g \in V \times C^{*}$, where we define $T_{r} g=g \cdot \gamma^{-1}$.

For instance a holomorphic representation $\rho$ of the complex Lie group $G_{H}(L)$ is a $G_{H}$-theta factor.

Given a $G_{H}$-theta factor $\tilde{J}$ of rank $m$, we define a holomorphic free action of $G_{H}(L)$ on the complex manifold $V \times C^{*} \times C^{m}$ by

$$
(g, \xi) \cdot \gamma=\left(g \gamma, \tilde{J}\left(\gamma^{-1}, g\right) \xi\right)
$$

where $g \in V \times C^{*}, \gamma \in G_{H}(L)$ and $\xi \in C^{m}$. The quotient space of $V \times C^{*}$ $\times C^{m}$ has a structure of a holomorphic vector bundle $F_{\tilde{J}}$ of rank $m$ over the complex torus $E$.

On the other hand, a theta factor $J$ of rank $m$ for the lattice $L$ is a map

$$
J: L \times V \rightarrow G L_{m}(C)
$$

such that

1) $J(\alpha, u)(\alpha \in L, u \in V)$ is holomorphic in $u$,

2) $J(\alpha+\beta, u)=J(\alpha, \beta+u) J(\beta, u)$

for $\alpha, \beta \in L$, and $u \in V$.

We define also a holomorphic free action of the lattice $L$ on $V \times C^{m}$ by

$$
(u, \xi) \alpha=(u+\alpha, J(\alpha, u) \xi),
$$


where $u \in V, \alpha \in L$ and $\xi \in C^{m}$.

The quotient of $V \times C^{m}$ is a holomorphic vector bundle $F_{J}$ of rank $m$ over the complex torus $E$. It is well-known that every holomorphic vector bundle over $E$ is obtained in this way.

LEMMA 3.1. Let $\tilde{J}$ be a $G_{H^{-}}$theta factor of rank $m$. For $\alpha \in L$ and $u \in V$ let

$$
J(\alpha, u)=\tilde{J}\left[\left(-\alpha, \varepsilon\left\{\frac{1}{2 i}(H(u, \alpha)+H(\alpha, \alpha))\right\}\right),(u, 1)\right] .
$$

Then $J$ is a theta factor for the lattice $L$ and the holomorphic vector bundles $F_{\tilde{J}}$ and $F_{J}$ are isomorphic.

Proof. We can verify readily that $J(\alpha, u)$ is a theta factor for $L$. We define a map $\psi$ from $V \times C^{m}$ into $V \times C^{*} \times C^{m}$ by

$$
\psi(u, \xi)=((u, 1), \xi)
$$

for all $u \in V$ and $\xi \in C^{m}$. We say that two points of $V \times C^{*} \times C^{m}$ are equivalent if they belong to the same orbit of $G_{H}(L)$. Two points $\psi(u, \xi)$ and $\psi(v, \eta)$ are equivalent if and only if there exists $(\alpha, \alpha) \in G_{H}(L)$ such that

$$
\left((u, 1)(\alpha, a), \tilde{J}\left[(\alpha, a)^{-1},(u, 1)\right] \xi\right)=((v, 1), \eta) .
$$

Since $(u, 1)(\alpha, a)=\left(u+\alpha, \varepsilon\left\{\frac{1}{2 i} H(u, \alpha)\right\} a\right)$, we get

$$
v=u+\alpha
$$

and

$$
a^{-1}=\varepsilon\left\{\frac{1}{2 i} H(u, \alpha)\right\}
$$

Then we get from (1.2) and (3.2), $(\alpha, \alpha)^{-1}=\left(-\alpha, \varepsilon\left\{\frac{1}{2 i}(H(u, \alpha)+H(\alpha, \alpha))\right\}\right)$ and so

$$
\eta=J(\alpha, u) \xi
$$

Thus if $\psi(u, \xi)$ and $\psi(v, \eta)$ are equivalent, then we have (3.1) and (3.3) and this means that $(u, \xi) \cdot \alpha=(v, \eta)$, that is, $(u, \xi)$ and $(v, \eta)$ are equivalent according to the action of $L$ on $V \times C^{*}$. 
Conversely let $(u, \xi) \alpha=(v, \eta)$, where $\alpha \in L$. Then defining $a$ by (3.2), we get $\psi(u, \xi)(\alpha, \alpha)=\psi(v, \eta)$. Hence we have shown that $\psi(u, \xi)$ and $\psi(v, \eta)$ are equivalent if and only if $(u, \xi)$ and $(v, \eta)$ are equivalent. Therefore $\psi$ defines an injective homomorphism $\bar{\psi}$ of $F_{J}$ into $F_{\tilde{J}}$. The homomorphism $\bar{\psi}$ is surjective. For, let $\left((u, a), \xi^{\prime}\right)$ be an arbitrary element of $V \times C^{*} \times C^{m}$. Then $\left(0, a^{-1}\right) \in G_{H}(L)$ and $\left((u, a), \xi^{\prime}\right)\left(0, a^{-1}\right)$ is of the form $((u, 1), \xi)$ and hence the orbit of $\left((u, a), \xi^{\prime}\right)$ contains an element of the form $\psi(u, \xi)$ and this proves that $\bar{\psi}$ is surjective.

As a special case of Lemma 3.1 we get the following Proposition 3.1 .

Proposition 3.1. Let $\rho$ be a holomorphic representation of the Heisenberg group $G_{H}(L)$. Then the holomorphic vector bundle $F_{\rho}$ over the complex torus $E=V / L$ associated with $\rho$ is isomorphic to the holomorphic vector bundle associated with the theta factor $J$ for the lattice $L$, where $J$ is given by

$$
J(\alpha, u)=\rho\left(0, \varepsilon\left\{\frac{1}{2 i} H(u, \alpha)+\frac{1}{4 i} H(\alpha, \alpha)\right\}\right) \Psi(\alpha),
$$

where

$$
\Psi(\alpha)=\rho\left(0, \varepsilon\left\{\frac{1}{4 i} H(\alpha, \alpha)\right\}\right) \rho(-\alpha, 1)
$$

From the definition of $\psi$ we get

$$
\Psi(\alpha+\beta)=\Psi(\alpha) \Psi(\beta) \rho\left(0, \varepsilon\left\{\frac{1}{2} A(\beta, \alpha)\right\}\right)
$$

for $\alpha, \beta \in L$.

THEOREM 3.1. Every holomorphic line bundle over $E=V / L$ is associated with a holomorphic representation of degree 1 of the Heisenberg group $G_{H}(L)$ for a suitable Hermitian form $H$.

Proof. A line bundle over $E$ is associated with a theta factor $j$ of rank 1 and we may assume that $j$ is in the normalized form (see [6]):

$$
j(\alpha, u)=\psi(\alpha) \varepsilon\left\{\frac{1}{2 i} H(u, \alpha)+\frac{1}{4 i} H(\alpha, \alpha)\right\},
$$

where $H$ is a Hermitian form on $V$ such that the imaginary part $A$ of 
$H$ is integral valued on $L$ and $\psi$ is a semi-character of $L$, that is, $\psi$ is a map $L \rightarrow C_{1}^{*}=\{z \in C|| z \mid=1\}$ such that $\psi(\alpha+\beta)=\psi(\alpha) \psi(\beta) \varepsilon\left\{\frac{1}{2} A(\alpha, \beta)\right\}$ for $\alpha, \beta \in L$. We define $\rho: G_{H}(L) \rightarrow C^{*}$ by

$$
\rho(\alpha, a)=\psi(-\alpha) \varepsilon\left\{-\frac{1}{4 i} H(\alpha, \alpha)\right\} a .
$$

It is easily verified that $\rho$ is a holomorphic representation of the Heisenberg group $G_{H}(L)$ of degree 1. It follows from (3.4) and (3.5) that the theta factor for $L$ corresponding to $\rho$ is precisely equal to $j$. Then the line bundle is isomorphic to the line bundle associated with the representation $\rho$ of $G_{H}(L)$.

\section{Holomorphic representations of $G_{H}(L)$ and $G_{A}(L)$.}

Let $H$ be a Hermitian form on $V$ and $A$ the imaginary part of $H$. Then $\varphi: G_{H} \rightarrow G_{A}$ defined by (2.2) induces an isomorphism of $G_{H}(L)$ onto $G_{A}(L)$. Hence there is a one-to-one correspondence between the set of holomorphic representations of $G_{A}(L)$ and that of $G_{H}(L)$. If $\rho_{A}$ is a holomorphic representation of $G_{A}(L)$, then $\rho_{H}=\rho_{A} \circ \varphi$ is the corresponding holomorphic representation of $G_{H}(L)$ and we have

$$
\rho_{H}(\alpha, a)=\rho_{A}\left(\alpha, \varepsilon\left\{-\frac{1}{4 i} H(\alpha, \alpha)\right\} \cdot a\right) .
$$

For instance, if $\psi: L \rightarrow C^{*}$ is a semi-character of $L$ (see the proof of theorem 3.1), then $\rho_{A}(\alpha, \alpha)=\psi(-\alpha) \cdot a$ defines a holomorphic representation of $G_{A}(L)$ and the corresponding representation $\rho_{H}$ of $G_{H}(L)$ is the one given by (3.6) in the proof of Theorem 3.1.

We remark here that an irreducible holomorphic representation $\sigma$ of the group $C^{*}$ is always of degree 1 and of the form

$$
\sigma(a)=a^{k}
$$

for all $a \in C^{*}$, where $k$ is an integer (cf. proof of Lemma 9.1 in $\S 9$ ).

Let $\rho$ be a holomorphic irreducible representation of $G_{A}(L)$. Then, since $\{0\} \times C^{*}$ is in the center of $G_{A}(L)$, every $\rho(0, a)$ is represented by a scalar operator by Schur's Lemma and hence

$$
\rho(0, a)=a^{k} \cdot 1
$$


for every $a \in C^{*}$, where $k$ is an integer and 1 is the identity operator. We call a holomorphic representation $\rho$ of $G_{A}(L)$ is homogeneous of order $k$ if the equation (4.2) holds for all $a \in C^{*}$. Every holomorphic irreducible representation of $G_{A}(L)$ is thus homogeneous.

Proposition 4.1. Suppose that the Heisenberg group $G_{A}(L)$ has a holomorphic homogeneous representation $\rho$ of order $k$ with $k \neq 0$. Then the alternating form $A$ is rational valued on the lattice $L$.

Proof. We have

$$
(\alpha, 1)(\beta, 1)=(\alpha+\beta, 1)\left(0, \varepsilon\left\{\frac{1}{2} A(\alpha, \beta)\right\}\right)
$$

and hence

$$
\rho(\alpha, 1) \rho(\beta, 1)=\rho(\alpha+\beta, 1) \varepsilon\left\{\frac{k}{2} A(\alpha, \beta)\right\}
$$

by (4.2). Then we get

$$
\operatorname{det} \rho(\alpha, 1) \cdot \operatorname{det} \rho(\beta, 1)(\operatorname{det} \rho(\alpha+\beta, 1))^{-1}=\varepsilon\left\{\frac{m k}{2} A(\alpha, \beta)\right\},
$$

where $m$ is the degree of $\rho$. Since the left hand side is symmetric in $\alpha$ and $\beta$ and $A$ is alternating, we should have $\varepsilon\{m k A(\alpha, \beta)\}=1$ for all $\alpha, \beta \in L$. Then $m k A(\alpha, \beta)$ is an integer for every $\alpha$ and $\beta$ in $L$ and $m k \neq 0$. Hence $A$ is rational valued on $L$.

We assume henceforth that $A$ is rational valued on $L$ and we always denote by $d$ the smallest positive integer such that $d A$ is integral valued on $L$.

We denote by $N$ the subgroup of $L$ consisting of all $\alpha \in L$ such that $A(\alpha, \beta)$ is an integer for every $\beta \in L$.

Then we have

$$
d L \subset N
$$

and hence $L / N$ is a finite abelian group of exponent $d$. In particular, $N$ is also a lattice of $V$. From the commutation rule (1.3) we get

$$
(\alpha, a)(\beta, b)=(\beta, b)(\alpha, a)(0, \varepsilon\{A(\alpha, \beta)\}) .
$$

It follows from this that $(\alpha, a)$ commutes with every $(\beta, b)$ if and only if $\alpha \in N$. Thus 


$$
G_{A}(N)=N \times C^{*}
$$

is the center of $G_{A}(L)$.

It follows then that $G_{A}(L)$ is abelian if and only if $N=L$. Now we have $N=L$ if and only if $A$ is integral valued on $L$. Thus we get that the Heisenberg group $G_{A}(L)$ and hence $G_{H}(L)$ is abelian if and only if $A$ is integral valued on the lattice $L$.

Let $\rho_{A}$ be a holomorphic homogeneous representation of $G_{A}(L)$ of order $k$ and $\rho_{H}$ the corresponding representation of $G_{H}(L)$. Then the theta factor $J$ corresponding to $\rho_{H}$ in Proposition 3.1 is of the form

$$
J(\alpha, u)=\varepsilon\left\{\frac{1}{2 i} k H(u, \alpha)+\frac{1}{4 i} k H(\alpha, \alpha)\right\} \varepsilon\left\{\frac{1}{4 i} k H(\alpha, \alpha)\right\} \rho_{H}(-\alpha, 1) .
$$

However we have

$$
\begin{aligned}
\rho_{H}(-\alpha, 1) & =\rho_{A}\left(-\alpha, \varepsilon\left\{-\frac{1}{4 i} H(\alpha, \alpha)\right\}\right) \\
& =\varepsilon\left\{-\frac{1}{4 i} k H(\alpha, \alpha)\right\} \rho_{A}(-\alpha, 1)
\end{aligned}
$$

and so we obtain

$$
J(\alpha, u)=\varepsilon\left\{\frac{1}{2 i} k H(u, \alpha)+\frac{1}{4 i} k H(\alpha, \alpha)\right\} \rho_{A}(-\alpha, 1) .
$$

\section{§5. Construction of irreducible representations of $G_{A}(L)$.}

In the sections 5 and 6 we denote by $A$ any $\boldsymbol{R}$-bilinear alternating form on a complex vector space $V$ of complex dimension $n$ which is rational valued on a lattice $L$ of $V$. We do not assume that $A$ is the imaginary part of an Hermitian form $H$. We denote by $d$ the smallest positive integer such that $d A$ is integral valued on the lattice $L$. There exists a basis

$$
\left\{\omega_{1}, \cdots, \omega_{n}, \omega_{1}^{\prime}, \cdots, \omega_{n}^{\prime}\right\}
$$

of $L$ such that $d A\left(\omega_{i}, \omega_{j}\right)=d A\left(\omega_{i}^{\prime}, \omega_{j}^{\prime}\right)=0$ and $d A\left(\omega_{i}, \omega_{j}^{\prime}\right)=e_{i} \delta_{i j}(i, j=1$, $\cdots, n)$, where $e_{1}, \cdots, e_{\ell}, e_{\ell+1}, \cdots, e_{n}$ are integers such that $e_{\ell+1}=\cdots=e_{n}$ $=0, e_{i}>0(1 \leqq i \leqq \ell), e_{i} \mid e_{i+1}(i=1,2, \cdots, \ell-1)$.

These integers are called the elementary divisors of the integral alternating form $d A$ on the lattice $L$. We have then 


$$
A\left(\omega_{i}, \omega_{j}^{\prime}\right)=\delta_{i j} e_{i} d^{-1}, \quad A\left(\omega_{i}, \omega_{j}\right)=A\left(\omega_{i}^{\prime}, \omega_{j}^{\prime}\right)=0 .
$$

Since $d$ is the smallest positive integer such that $d A$ is integral valued on $L$ and $e_{1} \mid e_{i}$ for all indices $i, d$ and $e_{1}$ have to be relatively prime. Let

$$
d_{i}=d\left(e_{i}, d\right)^{-1}, \quad(i=1,2, \cdots, \ell),
$$

where $\left(e_{i}, d\right)$ denotes the greatest common divisor of $e_{i}$ and $d$. Then $d_{i}$ is the smallest positive integer such that $d_{i} e_{i} d^{-1}$ is an integer and we have $d_{1}=d$ and $d_{i+1} \mid d_{i}(i=1,2, \cdots, \ell-1)$.

Let $N$ be the subgroup of $L$ consisting of all $\alpha \in L$ such that $A(\alpha, \beta)$ is an integer for every $\beta \in L$. Then

$$
\left\{d_{1} \omega_{1}, \cdots, d_{\ell} \omega_{\ell}, \omega_{\ell+1}, \cdots, \omega_{n}, d_{1} \omega_{1}^{\prime}, \cdots, d_{\ell} \omega_{\ell}^{\prime}, \omega_{\ell+1}^{\prime}, \cdots, \omega_{n}^{\prime}\right\}
$$

is a basis of $N$.

Let $L_{1}$ and $L_{1}^{\prime}$ be the subgroups of $L$ generated by $\left\{\omega_{1}, \cdots, \omega_{n}\right\}$ and $\left\{\omega_{1}^{\prime}, \cdots, \omega_{n}^{\prime}\right\}$ respectively and let

$$
N_{1}=L_{1} \cap N, \quad N_{2}^{\prime}=L_{2} \cap N .
$$

Then $N_{1}$ and $N_{2}^{\prime}$ are the subgroups of $N$ generated by

$$
\left\{d_{1} \omega_{1}, \cdots, d_{\ell} \omega_{\ell}, \omega_{\ell+1}, \cdots, \omega_{n}\right\} \quad \text { and } \quad\left\{d_{1} \omega_{1}^{\prime}, \cdots, d_{\ell} \omega_{\ell}^{\prime}, \omega_{\ell+1}^{\prime}, \cdots, \omega_{n}^{\prime}\right\}
$$

respectively and we have

$$
L=L_{1} \oplus L_{1}^{\prime}, \quad N=N_{1} \oplus N_{1}^{\prime}
$$

and

$$
L / N \cong L_{1} / N_{1} \oplus L_{1}^{\prime} / N_{1}^{\prime}
$$

Let

$$
K=L / N, \quad K_{1}=L_{1} / N_{1}, \quad K_{1}^{\prime}=L_{1}^{\prime} / N_{1}^{\prime} .
$$

Then $K, K_{1}$ and $K_{1}^{\prime}$ are finite abelian groups and we have

$$
K=K_{1} \oplus K_{1}^{\prime}, \quad K_{1} \cong K_{1}^{\prime}
$$

Let

$$
m=d_{1} d_{2}, \cdots, d_{\ell} .
$$

Then $m$ is the order of $K_{1}$ and $\left\{d_{1}, \cdots, d_{\ell}\right\}$ are the invariants of the 
finite abelian group $K_{1}$. We shall denote by $C\left(K_{1}\right)$ the vector space of all complex valued functions on $K_{1}$. Then $C\left(K_{1}\right)$ is an $m$-dimensional complex vector space. $C\left(K_{1}\right)$ is not only a vector space but also has an algebra structure and $C\left(K_{1}\right)$ is called the group algebra of the finite abelian group $K_{1}$. We shall denote by $R$ the regular representation of $K_{1}$. $\quad R$ is a representation of $K_{1}$ defined by

$$
(R(g) f)(h)=f(h+g)
$$

for all $g, h \in K_{1}$ and $f \in C\left(K_{1}\right)$.

For each $\lambda \in L$, we shall denote by $\bar{\lambda}$ the image of $\lambda$ in $K=L / N$. In particular if $\alpha \in L_{1}\left(\alpha^{\prime} \in L_{1}^{\prime}\right)$, then $\bar{\alpha}\left(\bar{\alpha}^{\prime}\right)$ belongs to $K_{1}\left(K_{1}^{\prime}\right)$. We now consider $\varepsilon\left\{A\left(\alpha, \alpha^{\prime}\right)\right\}$, where $\alpha \in L_{1}$ and $\alpha^{\prime} \in L_{1}^{\prime}$. We have $\varepsilon\left\{A\left(\alpha, \alpha^{\prime}\right)\right\}=\varepsilon\left\{A\left(\beta, \beta^{\prime}\right)\right\}$ whenever $\bar{\alpha}=\bar{\beta}$ and $\bar{\alpha}^{\prime}=\bar{\beta}^{\prime}$. For we have then $\alpha-\beta \in N$ and $\alpha^{\prime}-\beta^{\prime} \in N$ and hence $A\left(\alpha-\beta, \alpha^{\prime}\right)$ and $A\left(\beta, \alpha^{\prime}-\beta^{\prime}\right)$ are integers, whence

$$
\varepsilon\left(A\left(\alpha, \alpha^{\prime}\right)\right)=\varepsilon\left(A\left(\beta, \alpha^{\prime}\right)\right)=\varepsilon\left(A\left(\beta, \beta^{\prime}\right)\right) .
$$

Therefore we can define a pairing of abelian groups $K_{1}$ and $K_{1}^{\prime}$ by

$$
\left\langle g, g^{\prime}\right\rangle=\varepsilon\left\{A\left(\alpha, \alpha^{\prime}\right)\right\}, \quad g \in K_{1}, \quad g^{\prime} \in K_{1}^{\prime},
$$

where $g=\bar{\alpha}$ and $g^{\prime}=\bar{\alpha}^{\prime}$.

For any $g \in K_{1}$ and $g^{\prime} \in K_{1}^{\prime},\left\langle g, g^{\prime}\right\rangle$ is a $d$-th root of unity and we have

$$
\left\langle g+h, g^{\prime}\right\rangle=\left\langle g, g^{\prime}\right\rangle\left\langle h, g^{\prime}\right\rangle, \quad\left\langle g, g^{\prime}+h^{\prime}\right\rangle=\left\langle g, g^{\prime}\right\rangle\left\langle g, h^{\prime}\right\rangle .
$$

Then $\chi_{g^{\prime}}: g \rightarrow\left\langle g, g^{\prime}\right\rangle$ is a character of the abelian group $K_{1}$. Moreover, if $g^{\prime} \neq h^{\prime}$, we have $\chi_{g^{\prime}} \neq \chi_{h^{\prime}}$. For let $g^{\prime}=\bar{\alpha}^{\prime}, h^{\prime}=\bar{\beta}^{\prime}$ where $\alpha^{\prime}, \beta^{\prime} \in L_{1}^{\prime}$. If $\chi_{g^{\prime}}=\chi_{h^{\prime}}$, then we have $A\left(\alpha, \alpha^{\prime}-\beta^{\prime}\right) \in Z$ for all $\alpha \in L_{1}$ and hence $\alpha^{\prime}-\beta^{\prime}$ $\in N_{1}^{\prime}$. Then we get $\bar{\alpha}^{\prime}=\bar{\beta}^{\prime}$ and $g^{\prime}=h^{\prime}$. Since the order of $K_{1}^{\prime}$ is equal to the order of $K_{1}$ we can identify $K_{1}^{\prime}$ with the character group of $K_{1}$ by the identification map $g^{\prime} \rightarrow \chi_{g^{\prime}}$. The group $G_{A}(L)$ is identified with $L_{1} \times L_{1}^{\prime} \times C^{*}$ with the multiplication

$$
\left(\alpha, \alpha^{\prime}, \alpha\right)\left(\beta, \beta^{\prime}, b\right)=\left(\alpha+\beta, \alpha^{\prime}+\beta^{\prime}, \varepsilon\left\{\frac{1}{2}\left(A\left(\alpha, \beta^{\prime}\right)-A\left(\beta, \alpha^{\prime}\right)\right)\right\} a b\right) .
$$

Then $L_{1} \times\{0\} \times\{1\}$ and $\{0\} \times L_{1}^{\prime} \times\{1\}$ are subgroups isomorphic to $L_{1}$ and $L_{1}^{\prime}$ respectively and $L_{1} \times\{0\} \times C^{*}$ (resp. $\{0\} \times L_{1}^{\prime} \times C^{*}$ ) is also a subgroup isomorphic to the direct sum of $L_{1}$ and $C^{*}$ (resp. $L_{1}^{\prime}$ and $C^{*}$ ). 
For each integer $k$, let $\rho_{A}^{(k)}\left(\alpha, \alpha^{\prime}, a\right)$ be a linear transformation of $C\left(K_{1}\right)$ such that

$$
\left(\rho_{A}^{(k)}\left(\alpha, \alpha^{\prime}, a\right) f\right)(x)=a^{k} \varepsilon\left\{\frac{k}{2} A\left(\alpha, \alpha^{\prime}\right)+k A\left(\xi, \alpha^{\prime}\right)\right\} f(x+\bar{\alpha})
$$

for all $f \in C\left(K_{1}\right)$ and $x \in K_{1}$, where $\xi$ is an element of $L_{1}$ such that $\bar{\xi}=x$. Notice that if $\xi$ and $\eta$ are in $L_{1}$ and if $\bar{\xi}=\bar{\eta}$, then $\xi-\eta \in N$ and hence $\varepsilon\left\{A\left(\xi, \alpha^{\prime}\right)\right\}=\varepsilon\left\{A\left(\eta, \alpha^{\prime}\right)\right\}$. Hence the right hand side of (5.7) is independent of the choice of $\xi$ such that $\bar{\xi}=x$. It is easy to verify that $\rho_{A}^{(k)}$ is a holomorphic representation of $G_{A}(L)$ and $\rho_{A}^{(k)}$ is homogeneous of order $k$ in the sense defined in $\S 4$.

From (5.7) we get

$$
\begin{aligned}
& \rho_{A}^{(k)}(\nu, 0,1)=1, \quad \nu \in N_{1}, \\
& \rho_{A}^{(k)}\left(0, \nu^{\prime}, 1\right)=1, \quad \nu^{\prime} \in N_{1}^{\prime}, \\
& \rho_{A}^{(k)}(0,0, a)=a^{k} \cdot 1, \quad a \in C^{*},
\end{aligned}
$$

where 1 denotes the identity operator of $C\left(K_{1}\right)$.

THEOREM 5.1. Let $m_{k}$ be the order of the subgroup $k K_{1}$ of $K_{1}$ and $i_{k}$ the index of $k K_{1}$ in $K_{1}$. Then $\rho_{A}^{(k)}$ splits into a sum of $i_{k}$ inequivalent irreducible representations $\rho_{A, t}^{(k)}$ :

$$
\rho_{A}^{(k)}=\rho_{A, 1}^{(k)} \oplus \cdots \oplus \rho_{A, i_{k}}^{(k)} .
$$

The degree of each irreducible representation $\rho_{A, t}^{(k)}$ is $m_{k}$. The representation $\rho_{A}^{(k)}$ is irreducible if and only if $(k, d)=1$.

Proof. From the commutation rule

$$
(\alpha, 0,1)\left(0, \alpha^{\prime}, 1\right)=\left(0, \alpha^{\prime}, 1\right)(\alpha, 0,1)\left(0,0, \varepsilon\left\{A\left(\alpha, \alpha^{\prime}\right)\right\}\right)
$$

and from (5.5) and (5.8) we obtain

$$
\rho_{A}^{(k)}(\alpha, 0,1) \rho_{A}^{(k)}\left(0, \alpha^{\prime}, 1\right)=\left\langle\bar{\alpha}, k \bar{\alpha}^{\prime}\right\rangle \rho_{A}^{(k)}\left(0, \alpha^{\prime}, 1\right) \rho_{A}^{(k)}(\alpha, 0,1) .
$$

It also follows from (5.7) that

$$
\rho_{A}^{(k)}(\alpha, 0,1) f=R(\bar{\alpha}) f
$$

for all $f \in C\left(K_{1}\right)$ and $\alpha \in L_{1}$. Since $K_{1}$ is abelian, the regular representation $R$ decomposes into a sum of 1-dimensional representations and each 1-dimensional representation is a character of $K_{1}$. We know that 
the regular representation contains every irreducible representation and hence $R$ contains every characters of $K_{1}$. On the other hand we have identified $K_{1}^{\prime}$ with the character group of $K_{1}$ via the pairing (5.5). Hence $C\left(K_{1}\right)$ is a direct sum of 1-dimensional subspaces $W_{g^{\prime}}\left(g^{\prime} \in K_{1}^{\prime}\right)$ such that that $W_{g^{\prime}}$ consists of all $\phi \in C\left(K_{1}\right)$ with

$$
\rho_{A}^{(k)}(\alpha, 0,1) \phi=\left\langle\bar{\alpha}, g^{\prime}\right\rangle \phi
$$

for all $\alpha \in L_{1}$. Then we get from (5.9) that

$$
\rho_{A}^{(k)}\left(0, \alpha^{\prime}, 1\right) W_{g^{\prime}}=W_{g^{\prime}+k_{\alpha^{\prime}}} .
$$

Let $C_{1}, \cdots, C_{i_{k}}$ be distinct cosets of $K_{1}^{\prime}$ modulo $k K_{1}^{\prime}$ and let

$$
U_{t}=\sum_{g^{\prime} \in C_{t}} W_{g^{\prime}} \quad\left(1 \leqq t \leqq i_{k}\right) .
$$

The dimension of $U_{t}$ is equal to the order $m_{k}$ of $k K_{i}$, because $K_{1} \cong K_{1}^{\prime}$. Then $U_{t}$ is an invariant subspace of $C\left(K_{1}\right)$. We show that $U_{t}$ is irreducible. Let $U_{t}^{\prime}(\neq\{0\})$ be an invariant subspace of $U_{t}$. Then we have $\rho_{A}^{(k)}(\alpha, 0,1) U_{t}^{\prime}=U_{t}^{\prime}$ for all $\alpha \in L_{1}$ and hence there exists a $g^{\prime} \in C_{t}$ such that $W_{g^{\prime}} \subset U_{t}^{\prime}$. Then by (5.11) we get $W_{h^{\prime}} \subset U_{t}^{\prime}$ for all $h^{\prime} \in C_{t}$. Thus $U_{t}^{\prime}=U_{t}$ and $U_{t}$ is irreducible.

Let $\rho_{A, t}^{(k)}\left(\alpha, \alpha^{\prime}, a\right)$ be the restriction of $\rho_{A}^{(k)}\left(\alpha, \alpha^{\prime}, a\right)$ to $U_{t}$. Then $\rho_{A, t}^{(k)}$ is an irreducible representation of degree $m_{k}$ for each $t=1,2, \cdots, i_{k}$ and obviously $\rho_{A}^{(k)}$ decomposes into sum of these irreducible representations. If $\rho_{A, t}^{(k)}$ and $\rho_{A, s}^{(k)}$ were equivalent, the restriction of these representations to element of the form $(\alpha, 0,1)$ should yield the same set of characters of $K_{1}$ and if $t \neq s$, this is not the case. Hence $\rho_{A, t}^{(k)}$ are inequivalent irreducible representations.

The representation $\rho_{A}^{(k)}$ is irreducible if and only if $K_{1}=k K_{1} . \quad K_{1}$ is a direct sum of cyclic groups of order $d_{i}$ generated by $\bar{\omega}_{i}(i=1,2, \cdots, \ell)$ and we have $k K_{1}=K_{1}$ if and only if $\left(k, d_{i}\right)=1$ for $i=1,2, \cdots, \ell$. However $d=d_{1}$ and $d_{i} \mid d$ for $i=1,2, \cdots, \ell$. Hence we have $\left(k, d_{i}\right)=1$ for all $i$ if and only if $(k, d)=1$. This proves that $\rho_{A}^{(k)}$ is irreducible if and only if $k$ and $d$ are relatively prime.

If follows from (5.8) that $\rho_{A, t}^{(k)}$ is homogenous of order $k$ and $\rho_{A, t}^{(k)}(\nu, 0,1)=\rho_{A, t}^{(k)}\left(0, \nu^{\prime}, 1\right)=1, \nu \in N_{1}, \nu^{\prime} \in N_{1}^{\prime}$.

We now prove the following lemma:

LEMMA 5.1. Let $\rho$ be an irreducible representation of $G_{A}(L)$ in a 
complex vector space $U$ such that

$$
\rho(\nu, 0,1)=\rho\left(0, \nu^{\prime}, 1\right)=1 \quad \text { for } \nu \in N_{1}, \nu^{\prime} \in N_{1}^{\prime}
$$

and

$$
\rho(0,0, a)=a^{k} \cdot 1 \quad \text { for all } a \in C^{*} .
$$

Then $\rho$ is equivalent to one of the irreducible representations $\rho_{A, t}^{(k)}$.

Proof. By our condition on $\rho$, we can define a representation of $K_{1}$ by $\bar{\alpha} \rightarrow \rho(\alpha, 0,1)\left(\alpha \in L_{1}\right)$. We have also

$$
\rho(\alpha, 0,1) \rho\left(0, \alpha^{\prime}, 1\right)=\left\langle\bar{\alpha}, k \bar{\alpha}^{\prime}\right\rangle \rho\left(0, \alpha^{\prime}, 1\right\rangle \rho(\alpha, 0,1) .
$$

There exists $u \in U(u \neq 0)$ and $g^{\prime} \in K_{1}^{\prime}$ such that

$$
\rho(\alpha, 0,1)=\left\langle\bar{\alpha}, g^{\prime}\right\rangle u
$$

for all $\alpha \in L_{1}$. Let $u_{\beta^{\prime}}=\rho\left(0, \beta^{\prime}, 1\right) u$ for all $\bar{\beta}^{\prime} \in K_{1}^{\prime}$. Then we obtain from (5.12) that

$$
\rho(\alpha, 0,1) u_{\bar{\beta}^{\prime}}=\left\langle\bar{\alpha}, g^{\prime}+k \bar{\beta}^{\prime}\right\rangle u_{\bar{\beta}^{\prime}} .
$$

Let $C_{t}$ be the coset of $K_{1}^{\prime}$ modulo $k K_{1}^{\prime}$ containing $g^{\prime}$. Then there is an obvious linear map $T$ from $U_{t}=\sum_{g^{\prime} \in C_{t}} W_{g^{\prime}}$ onto the subspace of $U$ spanned by $\left\{u_{\beta}\right\}$ such that $T \circ \rho_{A, t}^{(k)}\left(\alpha, \alpha^{\prime}, a\right)=\rho\left(\alpha, \alpha^{\prime}, a\right) \circ T$ and as $\rho_{A, t}^{(k)}$ and $\rho$ are both irreducible, $T$ is bijective. Thus $\rho$ and $\rho_{A, t}^{(k)}$ are equivalent.

\section{§ 6. Classification of irreducible holomorphic representations of $G_{A}(L)$.}

THEOREM 6.1. Every irreducible holomorphic representation of $G_{A}(L)$ is equivalent to a representation of the form

$$
\sigma \otimes \rho_{A, t}^{(k)},
$$

where $\sigma$ is a 1-dimensional representation of $L$, that is, $\sigma$ is a 1-dimensional representation of $G_{A}(L)$ such that $\sigma(0, a)=1$ for all $a \in C^{*}$.

The following proof of Theorem 6.1 is due to J. Hano. The subgroup $N \times C^{*}$ is the center of $G_{A}(L)$ and hence

$$
\rho(\alpha, 1)=\rho^{\prime}(\alpha) \cdot 1
$$

for all $\alpha \in N$, where $\rho^{\prime}(\alpha) \in C^{*}$. The map $\rho^{\prime}: N \rightarrow C^{*}$ satisfies

$$
\rho^{\prime}(\alpha) \rho^{\prime}(\beta)=\rho^{\prime}(\alpha+\beta) \varepsilon\left\{\frac{1}{2} k A(\alpha, \beta)\right\}
$$


for $\alpha, \beta \in N$. We define a $R$-bilinear from $\Psi$ on $V \times V$ by

$$
\begin{gathered}
\Psi\left(\sum x_{k} \omega_{k}+\sum x_{k}^{\prime} \omega_{k}^{\prime}, \sum y_{k} \omega_{k}+\sum y_{k}^{\prime} \omega_{k}^{\prime}\right) \\
=\sum A\left(\omega_{k}, \omega_{k}^{\prime}\right) x_{k} y_{k}^{\prime} .
\end{gathered}
$$

Then $\Psi$ is a satellite form of $A$, namely $A(x, y)=\Psi(x, y)-\Psi(y, x)$ and letting $\psi_{0}(\alpha)=\varepsilon\left\{\frac{1}{2} k \Psi(\alpha, \alpha)\right\}, \alpha \in N$, we obtain a semi-character $\psi_{0}$ of $N$ with respect $k A$ which is integral valued on $N \times N$. That is, $\psi_{0}$ is a map from $N$ to $C_{1}^{*}=\{z \in C|| z \mid=1\}$ such that

$$
\psi_{0}(\alpha) \psi_{0}(\beta)=\psi_{0}(\alpha+\beta) \varepsilon\left\{\frac{1}{2} k A(\alpha, \beta)\right\} .
$$

From the definition of $\Psi$, it is clear that

$$
\psi_{0}(\alpha)=1
$$

if either $\alpha \in N_{1}$ or $\alpha \in N_{1}^{\prime}$.

There exists a 1-dimensional representation $\sigma^{\prime}$ of $N$ such that

$$
\rho^{\prime}(\alpha)=\sigma^{\prime}(\alpha) \psi_{0}(\alpha)
$$

for all $\alpha \in N$. We can extend $\sigma^{\prime}$ to a 1-dimensional representation $\sigma$ of L. Then we have $\left(\sigma^{-1} \otimes \rho\right)(\alpha, 1)=\sigma^{\prime}(\alpha)^{-1} \rho^{\prime}(\alpha) \cdot 1=\psi_{0}(\alpha) \cdot 1$ for all $\alpha \in N$ and hence $\left(\sigma^{-1} \otimes \rho\right)(\alpha, 1)=1$ for all $\alpha \in N_{1}$ and $\alpha \in N_{1}^{\prime}$. Then by Lemma $5.1, \sigma^{-1} \otimes \rho$ is equivalent to $\rho_{A, t}^{(k)}$ for some $t$ and hence $\rho$ is equivalent to $\sigma \otimes \rho_{A, t}^{(k)}$.

\$. Vector bundles defined by irreducible holomorphic representations of the group $G_{H}(L)$.

In this section we assume that $A$ is the imaginary part of an Hermitian form $H$ on $V$. Then there is a one-to-one correspondence between the set of irreducible holomorphic representations $\rho_{H}$ of $G_{H}(L)$ and the set of irreducible holomorphic representations $\rho_{A}$ of $G_{A}(L)$ and the one-to-one correspondence is given by (4.1), that is,

$$
\rho_{H}\left(\alpha, \alpha^{\prime}, a\right)=\rho_{A}\left(\alpha, \alpha^{\prime}, \varepsilon\left\{-\frac{1}{4 i} H\left(\alpha+\alpha^{\prime}, \alpha+\alpha^{\prime}\right)\right\} a\right) .
$$

We denote by $\rho_{H, t}^{(k)}$ the irreducible holomorphic representation of $G_{H}(L)$ which corresponds to the irreducible representation $\rho_{A, t}^{(k)}$ of $G_{A}(L)$ con- 
structed in $\S 5$.

Consider now the Hermitian form $k H$. The imaginary part of $k H$ is $k A$ and our decomposition $L=L_{1} \oplus L_{1}^{\prime}$ of $L$ which is defined by $A$ and which is used to define the representations $\rho_{A, t}^{(k)}$ is also a decomposition of $L$ with respect to the alternating form $k A$ (although the sublattice $N$ of $L$ defined by $A$ could be different from the one defined by $k A$ ). Therefore we can define the irreducible holomorphic representations $\rho_{k A, t}^{(j)}$ of the group $G_{k_{A}}(L)$ using the decomposition $L=L_{1} \oplus L_{1}^{\prime}$ and then the irreducible holomorphic representations $\rho_{k H, t}^{(j)}$ of the group $G_{k H}(L)$.

We prove the following lemma.

LEMMA 7.1. Let $F$ be the vector bundle over the complex torus $E=V / L$ defined by an irreducible holomorphic representation $\rho_{H, t}^{(k)}$ of the group $G_{H}(L)$. Then $F$ is isomorphic to a vector bundle $L_{\mu} \otimes F^{\prime}$, where the vector bundle $F^{\prime}$ is defined by the irreducible holomorphic representation $\rho_{k H}^{(1)}$ of the group $G_{k_{H}}(L)$ and $L_{\mu}$ is a line bundle defined by a 1-dimensional representation $\mu$ of $L$.

Proof. We have $\rho_{A, t}^{(k)}(0,0, a)=a^{k} \cdot 1$ and $\rho_{k A}^{(1)}(0,0, a)=a \cdot 1$. The theta factors $J$ and $J^{\prime}$ associated with the representations $\rho_{A, t}^{(k)}$ of $G_{A}(L)$ and with the representation $\rho_{k A}^{(1)}$ of $G_{k_{A}}(L)$ are then given by (4.3) and we have

$$
\begin{aligned}
J\left(\alpha+\alpha^{\prime}, u\right)= & \varepsilon\left\{\frac{1}{2 i} k H\left(u, \alpha+\alpha^{\prime}\right)+\frac{1}{4 i} k H\left(\alpha+\alpha^{\prime}, \alpha+\alpha^{\prime}\right)\right\} \\
& \cdot \rho_{A, t}^{(k)}\left(-\alpha,-\alpha^{\prime}, 1\right)
\end{aligned}
$$

and

$$
\begin{aligned}
J^{\prime}\left(\alpha+\alpha^{\prime}, u\right)= & \varepsilon\left\{\frac{1}{2 i} k H\left(u, \alpha+\alpha^{\prime}\right)+\frac{1}{4 i} k H\left(\alpha+\alpha^{\prime}, \alpha+\alpha^{\prime}\right)\right\} \\
& \cdot \rho_{k A}^{(1)}\left(-\alpha,-\alpha^{\prime}, 1\right) \quad \text { for } \alpha \in L_{1} \text { and } \alpha^{\prime} \in L_{1}^{\prime} .
\end{aligned}
$$

Let

$$
\tau\left(\alpha, \alpha^{\prime}, a\right)=\rho_{A, t}^{(k)}\left(\alpha, \alpha^{\prime}, 1\right) \cdot a
$$

for $\alpha \in L_{1}, \alpha^{\prime} \in L_{1}^{\prime}, a \in C^{*}$.

We show that $\tau$ is a representation of $G_{k A}(L)$. We have

$$
\begin{aligned}
& \tau\left(\alpha, \alpha^{\prime}, a\right) \tau\left(\beta, \beta^{\prime}, b\right) \\
& \quad=\rho_{A, t}^{(k)}\left(\left(\alpha, \alpha^{\prime}, 1\right)\left(\beta, \beta^{\prime}, 1\right)\right) a b
\end{aligned}
$$




$$
\begin{aligned}
& =\rho_{A, t}^{(k)}\left(\alpha+\beta, \alpha^{\prime}+\beta^{\prime}, \varepsilon\left\{\frac{1}{2} A\left(\alpha+\alpha^{\prime}, \beta+\beta^{\prime}\right)\right\}\right) a b \\
& =\rho_{A, t}^{(k)}\left(\alpha+\beta, \alpha^{\prime}+\beta^{\prime}, 1\right) \varepsilon\left\{\frac{1}{2} k A\left(\alpha+\alpha^{\prime}, \beta+\beta^{\prime}\right)\right\} a b .
\end{aligned}
$$

On the other hand we have

$$
\left(\alpha, \alpha^{\prime}, a\right)\left(\beta, \beta^{\prime}, b\right)=\left(\alpha+\beta, \alpha^{\prime}+\beta^{\prime}, \varepsilon\left\{\frac{1}{2} k A\left(\alpha+\alpha^{\prime}, \beta+\beta^{\prime}\right)\right\} a b\right)
$$

in the group $G_{k A}(L)$. Hence we have $\tau\left(\left(\alpha, \alpha^{\prime}, \alpha\right)\left(\beta, \beta^{\prime}, b\right)\right)=\rho_{A, t}^{(k)}\left(\alpha+\beta, \alpha^{\prime}+\right.$ $\left.\beta^{\prime}, 1\right) \varepsilon\left\{\frac{1}{2} k A\left(\alpha+\alpha^{\prime}, \beta+\beta^{\prime}\right)\right\} a b$ and we get $\tau\left(\alpha, \alpha^{\prime}, a\right) \tau\left(\beta, \beta^{\prime}, b\right)=\tau\left(\left(\alpha, \alpha^{\prime}, a\right)\right.$ $\left.\left(\beta, \beta^{\prime}, b\right)\right)$. Clearly $\tau$ is an irreducible holomorphic representation of $G_{k_{A}}(L)$ such that $\tau(0,0, \alpha)=a \cdot 1$. Then, by Theorem 6.1, we see that $\tau$ is equivalent to a representation $\mu \otimes \rho_{k A}^{(1)}$, where $\mu$ is a 1-dimensional representation of $L$. Since we have $\rho_{A, t}^{(k)}\left(\alpha, \alpha^{\prime}, 1\right)=\tau\left(\alpha, \alpha^{\prime}, 1\right)$, we see from (7.2) and (7.3) that the factor $J$ is equivalent to the factor $\mu \otimes J^{\prime}$. Let $L_{\mu}$ be the line bundle over $E$ associated with the representation $\mu$ of $L$. Then we have $F \cong L_{\mu} \otimes F^{\prime}$ and this proves Lemma 7.1.

THEOREM 7.1. Let $\rho$ be an irreducible holomorphic representation of $G_{H}(L)$ and $F_{\rho}$ the holomorphic vector bundle over $E=V / L$ defined by $\rho$. Then there exist a 1-dimensional representation $\sigma$ of $L$ such that

$$
F_{\rho} \cong L_{\sigma} \otimes F_{\rho_{k H}^{(1)}}
$$

where $k$ is the order of homogenuity of $\rho$ in the sense of $\S 4$ and $L_{\sigma}$ is the line bundle defined by $\sigma$.

Proof. By Theorem 6.1, $\rho$ is equivalent to a representation of $G_{H}(L)$ of the form $\eta \otimes \rho_{H, t}^{(k)}$ and hence $F_{\rho}$ is isomorphic to $L_{\eta} \otimes F$, where $F$ is defined by $\rho_{H, t}^{(k)}$. By Lemma 7.1, we have $F \cong L_{\mu} \otimes F_{\rho_{k H}^{(1)}}$, whence

$$
F_{\rho} \cong L_{\sigma} \otimes F_{\rho_{k H}^{(1)}} \quad \text { with } \quad \sigma=\eta \otimes \mu
$$

COROLLARY 7.2. Let $F$ be a holomorphic line bundle over $E=V / L$. Then $F \cong L_{\sigma} \otimes F_{\rho_{H}^{(1)}}$, where $\sigma$ is a 1-dimensional representation of $L$ and $H$ is a Hermitian form on $V$ whose imaginary part is integral valued on $L$. 
§ 8. Properties of the holomorphic vector bundle defined by the Schrödinger representation of $G_{H}(L)$.

According to Theorem 7.1, the study of holomorphic vector bundles associated with holomorphic irreducible representations of $G_{H}(L)$ for varying $H$ is reduced to the study of bundles associated with the irreducible representation $\rho_{H}^{(1)}$. In this and following section we shall denote the representations $\rho_{A}^{(1)}$ and $\rho_{H}^{(1)}$ by $D_{A}$ and $D_{H}$ respectively and call $D_{A}$ and $D_{H}$ the Schrödinger representation of $G_{A}(L)$ and $G_{H}(L)$. The representation $D_{A}$ is an irreducible holomorphic representation of $G_{A}(L)$ by Theorem 5.1 and it is defined by

$$
\left(D_{A}\left(\alpha, \alpha^{\prime}, a\right) f\right)(x)=a \cdot \varepsilon\left\{\frac{1}{2} A\left(\alpha, \alpha^{\prime}\right)+A\left(\xi, \alpha^{\prime}\right)\right\} f(x+\bar{\alpha})
$$

for every $f$ in the group algebra $C\left(K_{1}\right)$, where $\xi$ is any element in $L_{1}$ such that $\bar{\xi}=x \in K_{1}$. For each $g \in K_{1}$ let $f_{g}$ be the function on $K_{1}$ such that

$$
f_{g}(x)= \begin{cases}0, & x \neq g \\ 1, & x=g\end{cases}
$$

Then $\left\{f_{g}\right\}$ form a basis of $C\left(K_{1}\right)$ and we have

$$
f_{g}(x+h)=f_{g-h}(x)
$$

for any $g, h \in K_{1}$.

The function $x \rightarrow \varepsilon\left\{A\left(\xi, \alpha^{\prime}\right)\right\} f_{g}(x+\bar{\alpha})$ is equal to the function $\varepsilon\{A(\beta-$ $\left.\left.\alpha, \alpha^{\prime}\right)\right\} f_{g-\alpha}$ where $\bar{\xi}=x$ and $\bar{\beta}=g$. Then we get from (8.1) and (8.2) the following formula:

$$
D_{A}\left(\alpha, \alpha^{\prime}, a\right) f_{g}=a \cdot \varepsilon\left\{-\frac{1}{2} A\left(\alpha, \alpha^{\prime}\right)+A\left(\beta, \alpha^{\prime}\right)\right\} f_{g-\alpha},
$$

where $\beta$ is any element in $L_{1}$ such that $\bar{\beta}=g$.

The theta factor $J_{H}$ associated with $D_{H}$ is given by (4.3) and we get

$$
J_{H}\left(\alpha+\alpha^{\prime}, u\right)=\varepsilon\left\{\frac{1}{2 i} H\left(u, \alpha+\alpha^{\prime}\right)+\frac{1}{4 i} H\left(\alpha+\alpha^{\prime}, \alpha+\alpha^{\prime}\right)\right\} D_{A}\left(-\alpha,-\alpha^{\prime}, 1\right)
$$

and we obtain from (8.3): 


$$
\begin{aligned}
J_{H}(\alpha+ & \left.\alpha^{\prime}, u\right) f_{g} \\
= & \varepsilon\left\{\frac{1}{2 i} H\left(u, \alpha+\alpha^{\prime}\right)+\frac{1}{4 i} H\left(\alpha+\alpha^{\prime}, \alpha+\alpha^{\prime}\right)\right\} \\
& \cdot \varepsilon\left\{-\frac{1}{2} A\left(\alpha, \alpha^{\prime}\right)-A\left(\beta, \alpha^{\prime}\right)\right\} f_{g+\bar{\alpha}},
\end{aligned}
$$

where $g=\bar{\beta}, \beta \in L_{1}$ and $\alpha \in L_{1}, \alpha^{\prime} \in L_{1}^{\prime}$.

In particular we have

$$
J_{H}(\alpha, u) f_{g}=\varepsilon\left\{\frac{1}{2 i} H(u, \alpha)+\frac{1}{4 i} H(\alpha, \alpha)\right\} f_{g+\alpha} \quad\left(\alpha \in L_{1}\right)
$$

and

$$
J_{H}\left(\alpha^{\prime}, u\right) f_{g}=\varepsilon\left\{\frac{1}{2 i} H\left(u, \alpha^{\prime}\right)+\frac{1}{4 i} H\left(\alpha^{\prime}, \alpha^{\prime}\right)\right\} \varepsilon\left\{-A\left(\beta, \alpha^{\prime}\right)\right\} f_{g}, \quad\left(\alpha^{\prime} \in L_{1}^{\prime}\right)
$$

Let

$$
M=N_{1} \oplus L_{1}^{\prime}
$$

Then $M$ is a lattice of $V$ containing $N=N_{1} \oplus N_{1}^{\prime}$ and contained in $L=$ $L_{1} \oplus L_{1}^{\prime}$ and $A$ is integral valued on $M$.

Let

$$
E_{M}=V / M
$$

Then $E_{M}$ is a complex torus and there is a homomorphism

$$
\varphi: E_{M} \rightarrow E=V / L
$$

and the kernel of $\varphi$ is $L / M$ which we identify canonically with $K_{1}$.

For each $g \in K_{1}$ let

$$
\psi_{g}\left(\nu+\alpha^{\prime}\right)=\varepsilon\left\{-\frac{1}{2} A\left(\nu, \alpha^{\prime}\right)-A\left(\beta, \alpha^{\prime}\right)\right\} \quad\left(g=\bar{\beta}, \beta \in L_{1}\right)
$$

for all $\nu+\alpha^{\prime} \in M$ with $\nu \in N_{1}$ and $\alpha^{\prime} \in L_{1}^{\prime}$. Then $\psi_{g}$ is a semi-character on the lattice $M$, i.e. we have

$$
\psi_{g}\left(m+m^{\prime}\right)=\psi_{g}(m) \psi_{g}\left(m^{\prime}\right) \varepsilon\left\{\frac{1}{2} A\left(m^{\prime}, m\right)\right\}
$$

for all $m, m^{\prime} \in M$. 
Let

$$
\left.j_{g}\left(\nu+\alpha^{\prime}, u\right)=\psi_{g}\left(\nu+\alpha^{\prime}\right) \varepsilon\left\{\frac{1}{2 i} \dot{H(u, \nu}+\alpha^{\prime}\right)+\frac{1}{4 i} H\left(\nu+\alpha^{\prime}, \nu+\alpha^{\prime}\right)\right\} .
$$

Since $\bar{\nu}=0$ (the zero element of $K_{1}$ ) for $\nu \in N_{1}$, we get from (8.4) that

$$
J_{H}\left(\nu+\alpha^{\prime}, u\right) f_{g}=j_{g}\left(\nu+\alpha^{\prime}, u\right) f_{g}
$$

for all $g \in K_{1}, \nu \in N_{1}, \alpha^{\prime} \in L_{1}^{\prime}$. Then we conclude that $j_{g}$ is a theta factor for the lattice $M$ and that $J_{H}\left(\nu+\alpha^{\prime}, u\right)$ is the diagonal matrix whose diagonal entries are $j_{g}\left(\nu+\alpha^{\prime}, u\right)$.

Let $L_{g}\left(g \in K_{1}\right)$ be the line bundle over $E_{M}$ defined by the theta factor $j_{g}$. Now $J_{H}(m, u)(m \in M)$ is a theta factor for the lattice $M$ and the vector bundle over $E_{M}$ defined by this factor is the pull back $\varphi^{*} F_{D_{H}}$, where $F_{D_{H}}$ is the vector bundle over $E$ associated with the Schrödinger representation $D_{H}$.

Then we have

$$
\varphi^{*} F_{D_{H}}=\sum_{g \in K_{1}} L_{g}
$$

If $g \neq h$, then $L_{g}$ and $L_{h}$ are not isomorphic. For we have

$$
j_{g}\left(\nu+\alpha^{\prime}, u\right)=\varepsilon\left\{-A\left(\beta-\gamma, \alpha^{\prime}\right)\right\} j_{h}\left(\nu+\alpha^{\prime}, u\right)
$$

where $\bar{\beta}=g$ and $\bar{\gamma}=h$, and the representation of $M$ defined by $\nu+\alpha^{\prime}$ $\rightarrow \varepsilon\left\{-A\left(\beta-\gamma, \alpha^{\prime}\right)\right\}$ is not trivial except for the case $\bar{\beta}=\bar{\gamma}$. Thus two normalized factors $j_{g}$ and $j_{h}$ for the lattice $M$ are distinct and hence $L_{g}$ and $L_{h}$ are not isomorphic. However they are algebraical equivalent, namely they have the same Chern class (see [6]).

Let now

$$
T_{g}: E_{M} \rightarrow E_{M}
$$

be the translation of the complex torus $E_{M}$ by an element $g \in K_{1}\left(\subset E_{M}\right)$. We show that

$$
T_{g}^{*} L_{h} \cong L_{h-g},
$$

where $T_{g}^{*} L_{h}$ denotes the pull-back of $L_{h}$ by $T_{g}$. The pull-back $T_{g}^{*} L_{h}$ is defined by the factor 


$$
\varepsilon\left\{\frac{1}{2 i} H\left(\beta, \nu+\alpha^{\prime}\right)\right\} j_{h}\left(\nu+\alpha^{\prime}, u\right)
$$

where $\bar{\beta}=g$. However

$$
\frac{1}{2 i} H\left(\beta, \nu+\alpha^{\prime}\right)=A\left(\beta, \nu+\alpha^{\prime}\right)+\frac{1}{2 i} H\left(\nu+\alpha^{\prime}, \beta\right)
$$

and $\varepsilon\left\{A\left(\beta, \nu+\alpha^{\prime}\right)\right\}=\varepsilon\left\{A\left(\beta, \alpha^{\prime}\right)\right\}$. Hence $\varepsilon\left\{\frac{1}{2 i} H\left(\beta, \nu+\alpha^{\prime}\right)\right\}=\varepsilon\left\{A\left(\beta, \alpha^{\prime}\right)\right\}$ $\varepsilon\left\{\frac{1}{2 i} H\left(\nu+\alpha^{\prime}, \beta\right)\right\}$. We have also $\varepsilon\left\{\frac{1}{2 i} H\left(\nu+\alpha^{\prime}, \beta\right)\right\}=P\left(u+\nu+\alpha^{\prime}\right) / P(u)$, where $P(u)=\varepsilon\left\{\frac{1}{2 i} H(u, \beta)\right\}$. Thus

$$
\varepsilon\left\{\frac{1}{2 i} H\left(\beta, \nu+\alpha^{\prime}\right)\right\}=\varepsilon\left\{A\left(\beta, \alpha^{\prime}\right)\right\} P\left(u+\nu+\alpha^{\prime}\right) / P(u)
$$

and since $P(u)$ is a non-vanishing holomorphic function on $V$, $P\left(u+\nu+\alpha^{\prime}\right) / P(u)$ is a trivial factor. Thus $T_{g}^{*} L_{h}$ is isomorphic to the line bundle defined by the factor $\varepsilon\left\{A\left(\beta, \alpha^{\prime}\right)\right\} \cdot j_{h}\left(\nu+\alpha^{\prime}, u\right)$ which is equal to $j_{h-g}$ by (8.13). Thus $T_{g}^{*} L_{h} \cong L_{h-g}$.

Summing up we obtain the following theorem.

THEOREM 8.1. Let $F_{D_{H}}$ be the holomorphic vector bundle on the complex torus $E$, associated with the Schrödinger representation $D_{H}$ of $G_{H}(L)$. There exist a complex torus $E_{M}$ and a homomorphism $\varphi: E_{M} \rightarrow$ $E$ of $E_{M}$ onto $E$ whose kernel is $K_{1}$ and there are holomorphic line bundles $\left\{L_{g}\right\}_{g \in R_{1}}$ on $E_{M}$ such that $L_{g} \nRightarrow L_{h}$ for $g \neq h, T_{g}^{*} L_{h}=L_{h-g}$ and $\varphi^{*} F_{D_{H}} \cong \sum_{g \in K_{1}} L_{g}$.

Remark. Theorem 8.1 shows that $F_{D_{H}}$ is the direct images $\varphi_{*} L_{g}$ of any one of line bundles $L_{g}$ on $E_{M}$.

We consider now the vector bundle End $\left(F_{D_{H}}\right)$. Then there exists an exact sequence

$$
0 \rightarrow I \rightarrow \text { End }\left(F_{D_{H}}\right) \rightarrow Q \rightarrow 0,
$$

where $I$ is the trivial line bundle and $Q$ is the quotient bundle. The homomorphism $I \rightarrow$ End $\left(F_{D_{H}}\right)$ is defined by associating to each complex number $c$ the multiplication of each fibre of $F_{D_{H}}$ by $c$. We get then a homomorphism of cohomologies 


$$
H^{j}(E, \mathcal{O}) \rightarrow H^{j}\left(E, \text { End }\left(F_{D_{H}}\right)\right)
$$

induced by $I \rightarrow F_{D_{H}}$.

We have the following theorem due to Oda [5].

THEOREM 8.2 (Oda). Let $F$ be a holomorphic vector bundle on a complex torus $X$. Then the following two statements are equivalent.

(1) There exist a complex torus $Y$ and a homomorphism $\varphi$ of $Y$ onto $X$ and a line bundle $L$ on $Y$ such that

$$
T_{g}^{*} L \not L L \quad \text { for all } g \in \operatorname{ker}(\varphi) \text { and } \varphi_{*} L=F .
$$

(2) The homomorphism $H^{j}(X, \mathcal{O}) \rightarrow H^{j}(X$, End $(F))$ induced by $I \rightarrow$ End $(F)$ is an isomorphism for all $j$.

Applying the theorem of Oda for the vector bundle $F_{D_{H}}$, we get from Theorem 8.1 the following corollary.

COROLLARY 8.2. We have

$$
H^{j}(E, \mathcal{O}) \cong H^{j}\left(E, \text { End }\left(F_{D_{H}}\right)\right)
$$

for all $j$. In particular we have

$$
\Gamma\left(E, \text { End }\left(F_{D_{H}}\right)\right)=C .
$$

A vector bundle $F$ is said to be simple if $\Gamma(E$, End $(F))=C$. A simple vector bundle is indecomposable. Hence

COROLLARY 8.3. The vector bundle $F_{D_{H}}$ is simple and hence it is indecomposable.

Moreover we have $H^{j}(E, \mathcal{O}) \cong H^{0, j}(E, C)$ and since $E$ is an $n$-dimensional complex torus, $\operatorname{dim} H^{0, j}(E, C)=\left(\begin{array}{c}n \\ j\end{array}\right)$. Thus we get

$$
\operatorname{dim} H^{j}\left(E, \text { End }\left(F_{D_{H}}\right)\right)=\left(\begin{array}{c}
n \\
j
\end{array}\right)
$$

We now consider a theta function $\theta$ for the factor $J_{H}$. The function $\theta$ is a $C\left(K_{1}\right)$-valued holomorphic function on $V$ satisfying the equation

$$
\theta\left(\alpha+\alpha^{\prime}+u\right)=J_{H}\left(\alpha+\alpha^{\prime}, u\right) \theta(u)
$$

for all $\alpha \in L_{1}, \alpha^{\prime} \in L_{1}^{\prime}$ and $u \in V$.

We write 


$$
\theta(u)=\sum_{g \in K_{1}} \theta_{g}(u) f_{g}
$$

and we identify $\theta(u)$ with the column vector $\left(\theta_{g}(u)\right)$ of holomorphic functions $\theta_{g}(u)$ on $V$. We see from (8.4) that the components of the vector $\theta$ satisfy the following equation.

$$
\begin{aligned}
\theta_{g}\left(\alpha+\alpha^{\prime}+u\right) \\
=\varepsilon \\
\left.\varepsilon \frac{1}{2 i} H\left(u, \alpha+\alpha^{\prime}\right)+\frac{1}{4 i} H\left(\alpha+\alpha^{\prime}, \alpha+\alpha^{\prime}\right)\right\} \\
\cdot \varepsilon\left\{\frac{1}{2} A\left(\alpha, \alpha^{\prime}\right)-A\left(\beta, \alpha^{\prime}\right)\right\} \theta_{g-\alpha}(u),
\end{aligned}
$$

where $\bar{\beta}=g$.

If $\nu \in N_{1}$, then $A\left(\nu, \alpha^{\prime}\right)$ is an integer and $\varepsilon\left\{\frac{1}{2} A\left(\nu, \alpha^{\prime}\right)\right\}= \pm 1$ and hence $\varepsilon\left\{\frac{1}{2} A\left(\nu, \alpha^{\prime}\right)\right\}=\varepsilon\left\{-\frac{1}{2} A\left(\nu, \alpha^{\prime}\right)\right\}$. Then we get from (8.10) that

$$
\theta_{g}\left(\nu+\alpha^{\prime}+u\right)=j_{g}\left(\nu+\alpha^{\prime}, u\right) \theta_{g}(u)
$$

for $\nu \in N_{1}, \alpha^{\prime} \in L_{1}^{\prime}$. This shows that the component $\theta_{g}(u)$ is a theta function for the lattice $M$ and the factor $j_{g}$. In particular $\theta_{0}(0$ is the zero element of $K_{1}$ ) is a theta function for the lattice $M$ and the factor $j_{0}$. Letting $\alpha=\beta, \alpha^{\prime}=0$ and replacing $u$ by $-\beta+u$ in (8.14) we get

$$
\theta_{g}(u)=\varepsilon\left\{\frac{1}{2 i} H(u, \beta)-\frac{1}{4 i} H(\beta, \beta)\right\} \theta_{0}(-\beta+u)
$$

where $\bar{\beta}=g$. This shows that $\theta_{g}$ is uniquely determined by $\theta_{0}$.

Let $h$ be a theta function for the lattice $M$ and the factor $j_{0}$. We show that there exists a theta function $\theta$ for the lattice $L$ and the factor $J_{H}$ such that $h=\theta_{0}$. To see this we first assert that

$$
\begin{aligned}
& \varepsilon\left\{\frac{1}{2 i} H(u, \beta)-\frac{1}{4 i} H(\beta, \beta)\right\} h(-\beta+u) \\
& =\varepsilon\left\{\frac{1}{2 i} H(u, \gamma)-\frac{1}{4 i} H(\gamma, \gamma)\right\} h(-\gamma+u)
\end{aligned}
$$

for any two elements $\beta$ and $\gamma$ in $L_{1}$ such that $\bar{\beta}=\bar{\gamma}$. In fact, if $\bar{\beta}=\bar{\gamma}$, then we have $\nu=\beta-\gamma \in N_{1}$ and hence

$$
h(-\gamma+u)=h(\nu-\beta+u)=j_{0}(\nu,-\beta+u) h(-\beta+u) .
$$


We have

$$
\begin{aligned}
j_{0}(\nu,-\beta+u)= & \varepsilon\left\{\frac{1}{2 i} H(u-\beta, \beta-\gamma)+\frac{1}{4 i} H(\beta-\gamma, \beta-\gamma)\right\} \\
= & \varepsilon\left\{\frac{1}{2 i} H(u, \beta)-\frac{1}{4 i} H(\beta, \beta)\right\} \varepsilon\left\{-\frac{1}{2 i} H(u, \gamma)+\frac{1}{4 i} H(\gamma, \gamma)\right\} \\
& \cdot \varepsilon\left\{\frac{1}{4 i}(H(\beta, \gamma)-H(\gamma, \beta)\} .\right.
\end{aligned}
$$

However, as $A(\beta, \gamma)=0$, we have $H(\beta, \gamma)=H(\gamma, \beta)$, whence

$$
\varepsilon\left\{\frac{1}{2 i} H(u, \gamma)-\frac{1}{4 i} H(\gamma, \gamma)\right\} j_{0}(\nu,-\beta+u)=\varepsilon\left\{\frac{1}{2 i} H(u, \beta)-\frac{1}{4 i} H(\beta, \beta)\right\}
$$

from which our assertion follows.

We can then define $\theta_{g}$ for each $g \in K_{1}$ by

$$
\theta_{g}(u)=\varepsilon\left\{\frac{1}{2 i} H(u, \beta)-\frac{1}{4 i} H(\beta, \beta)\right\} h(-\beta+u)
$$

where $\beta$ is any element in $L_{1}$ such that $\bar{\beta}=g$. In particular letting $\beta=0$ we have $\theta_{0}=h$. Then we can verify easily that the vector $\left(\theta_{g}\right)$ satisfy the equation (8.14). Thus $\theta=\left(\theta_{g}\right)$ is a theta function for the lattice $L$ and the factor $J_{H}$ such that $\theta_{0}=h$.

We have shown that $h \rightarrow \theta$ defines a bijective map from the vector space of all theta functions for $M$ and $j_{0}$, which is identified with the vector space $\Gamma\left(E_{M}, L_{0}\right)$ of all holomorphic sections of the line bundle $L_{0}$, onto the vector space of all theta functions for $L$ and $J_{H}$. The latter vector space is identified with the vector space $\Gamma\left(E, F_{D_{H}}\right)$ of all holomorphic sections of the vector bundle $F_{D_{H}}$.

Since the theta factor $j_{0}$ is in the normalized form (8.10), we know that if $\Gamma\left(E_{M}, L_{0}\right) \neq\{0\}$, then the Hermitian form $H$ is positive ( $\left.\geqq\right)$ and $\operatorname{dim} \Gamma\left(E_{M}, L_{0}\right)$ is equal to the reduced Pfaffian of $A$ relative to $M$, that is, the product of nonzero elementary divisors of the integral alternating form $A$ on $M$ (see [1], [4], [6]).

The lattice $M$ has a basis

$$
\left\{d_{1} \omega_{1}, d_{2} \omega_{2}, \cdots, d_{\ell} \omega_{\ell}, \omega_{\ell+1}, \cdots, \cdots, \omega_{n}, \omega_{1}^{\prime}, \cdots, \omega_{n}^{\prime}\right\}
$$

and we have

$$
A\left(\omega_{i}, \omega_{j}\right)=A\left(\omega_{i}^{\prime}, \omega_{j}^{\prime}\right)=0
$$




$$
A\left(\omega_{i}, \omega_{j}^{\prime}\right)=\frac{e_{i}}{d} \delta_{i j}
$$

where $e_{\ell+1}=\cdots=e_{n}=0$ and $e_{1}, \cdots, e_{\ell}$ are non-zero (see $\S 5$ ). Then

$$
A\left(d_{i} \omega_{i}, \omega_{i}^{\prime}\right)=e_{i} \cdot d_{i} d^{-1}
$$

and by (5.1) we get

$$
A\left(d_{i} \omega_{i}, \omega_{i}^{\prime}\right)=e_{i}\left(e_{i}, d\right)^{-1}
$$

for $i=1,2, \cdots, \ell$. Therefore $e_{i}\left(e_{i}, d\right)^{-1}(i=1, \cdots, \ell)$ are non-zero elementary divisors of the integral alternating form $A$ on $M$ and we get

$$
\operatorname{dim} \Gamma\left(E_{M}, L_{0}\right)=\operatorname{dim} \Gamma\left(E, F_{D_{H}}\right)=\prod_{i=1}^{\ell} e_{i}\left(e_{i}, d\right)^{-1} .
$$

Summing up we get

THEOREM 8.3. Let $F_{D_{H}}$ be the holomorphic vector bundle over $E$ defined by the Schrödinger representation of the group $G_{H}(L)$. The rank of $F_{D_{H}}$ is equal to $d^{\ell} \prod_{i=1}^{\ell}\left(e_{i}, d\right)^{-1}$ and the dimension of the vector space $\Gamma\left(E, F_{D_{H}}\right)$ of holomorphic sections of $F_{D_{H}}$ is nonzero if and only if $H$ is positive and $H \neq 0$ and if this is the case, we have

$$
\operatorname{dim} \Gamma\left(E, F_{D_{H}}\right)=\prod_{i=1}^{\ell} e_{i}\left(e_{i}, d\right)^{-1}
$$

here $d$ is the smallest positive integer such that $d A$ is integral valued on $L, A$ being the imaginary part of $H$, and $e_{1}, \cdots, e_{\ell}$ denote the nonzero elementary divisors of the integral alternating form $d A$ on $L$.

For any complex torus $E=V / L$ and a theta factor $J$ of rank $r$ for $L$, let us denote by $D^{p}(E, J)$ the complex vector space of all $C^{r}$-valued differential form $\omega$ of type $(0, p)$ on $V$ such that

$$
\left(T_{\alpha}^{*} \omega\right)_{u}=J(\alpha, u) \omega_{u}
$$

for every $\alpha \in L$ and $u \in V$, where $T_{\alpha}: V \rightarrow V$ denotes the translation of $V$ by $\alpha$ and $\omega_{u}$ is the value of $\omega$ at $u$. Since $J(\alpha, u)$ is holomorphic in $u$, we have $d^{\prime \prime} \omega \in D^{p+1}(E, J)$ for every $\omega \in D^{p}(E, J)$. Thus $D(E, J)=$ $\sum_{p} D^{p}(E, J)$ is a complex with coboundary operator $d^{\prime \prime}$ and the cohomology groups $H^{p}(E, J)$ of this complex is canonically identified with the 
cohomology groups $H^{p}\left(E, F_{J}\right)$ of the sheaf of germs of holomorphic sections of the vector bundle $F_{J}$ defined by $J$.

Consider now $D^{p}\left(E, J_{D_{H}}\right)$ and let $\omega \in D^{p}\left(E, J_{D_{H}}\right)$. We can represent $\omega$ by a column vector $\left(\omega_{g}\right)\left(g \in K_{1}\right)$ and we can show precisely as in the proof of Theorem 8.3 that $\omega_{0}$ is an element of $D^{p}\left(E_{M}, j_{0}\right)$ and that the map from $D^{p}\left(E_{M}, j_{0}\right)$ to $D^{p}\left(E, J_{D_{H}}\right)$ defined by $\omega_{0} \rightarrow \omega$ is a bijective linear map for each $p$ commuting with $d^{\prime \prime}$. Thus this induces an isomorphism of cohomologies

$$
H^{p}\left(E_{M}, L_{0}\right) \cong H^{p}\left(E, F_{D_{H}}\right)
$$

for all $p$.

We now have the following theorem of Mumford [4] (see also [7]). Let $E$ be a complex torus and $F$ a line bundle over $E$ defined by a normalized theta factor $\psi(\alpha)\left\{\frac{1}{2 i} H(u, \alpha)+\frac{1}{4 i} H(\alpha, \alpha)\right\}$, where $\alpha \in L, u \in V$ with $E=V / L$. Assume that the Hermitian form $H$ is non-degenerate and let $i(H)$ be the number of negative eigenvalues of $H$. Then $H^{p}(E, F)=0$ except for $p=i(H)$ and the dimension of $H^{i(H)}(E, F)$ is equal to the Plaffian of the imaginary part $A$ of $H$.

Applying this theorem of Mumford to the line bundle $L_{0}$ over $E_{M}$ we obtain from the isomorphism (8.17) the following theorem.

THEOREM 8.4. The notation being as in Theorem 8.3, we assume that $H$ is non-degenerate and let $i(H)$ denote the number of negative eigenvalues of $H$. Then we have

$$
H^{p}\left(E, F_{D_{H}}\right)=0, \quad p \neq i(H)
$$

and

$$
\operatorname{dim} H^{i(H)}\left(E, F_{D_{H}}\right)=\prod_{i=1}^{n} e_{i}\left(e_{i}, d\right)^{-1}
$$

\section{§9. Vector bundles associated with indecomposable holomorphic representa-} tions of $G_{H}(L)$, their tensor products and Chern classes.

In $\S 4$ we called a holomorphic representation $\rho$ of $G_{A}(L)$ is homogeneous of order $k$, if $\rho(0, a)=a^{k} \cdot 1$ for all $a \in C^{*}$, where $k$ is an integer. Every irreducible holomorphic representation is homogeneous and every representation of $L$ is regarded as a homogeneous representation of degree 0 . 
LEMMA 9.1. Let $\rho$ be a holomorphic representation of $G_{A}(L)$. Then $\rho$ splits into a sum of holomorphic homogeneous representations $\rho_{1}, \cdots$, $\rho_{s}$ having distinct orders.

As a corollary we get

COROLlaRY 9.1. Every indecomposable holomorphic representation of $G_{A}(L)$ is homogeneous.

To prove Lemma 9.1 we first observe that every holomorphic representation $\tilde{\sigma}$ of $C$ is of the form

$$
\tilde{\sigma}(z)=\exp z B
$$

where $B$ is a complex matrix. Now let $\sigma: C^{*} \rightarrow G L_{r}(C)$ be a holomorphic representation and let $p: C \rightarrow C^{*}$ be the covering homomorphism defined by $p(z)=\exp z$. Then $\operatorname{ker} p=\{2 \pi i m \mid m \in Z\}$. We can lift $\sigma$ be a representations $\tilde{\sigma}: C \rightarrow G L_{r}(C)$ such that $\tilde{\sigma}(z)=\sigma(p(z))$. There is a $r \times r$ complex matrix $B$ such that $\tilde{\sigma}(z)=\exp z B$. Then we have $1=\sigma(p(2 \pi i))$ $=\exp 2 \pi i B$. Then $t \rightarrow \exp 2 \pi i t B(t \in R)$ defines a representation of $R /(1)$ and hence we may assume that $\exp 2 \pi i t B$ are unitary for all $t \in \boldsymbol{R}$. Then $i B$ is skew Hermitian and so $B$ is a Hermitian matrix. Again we may assume that $B$ is diagonal. Then as $\exp 2 \pi i B=1$, the diagonal entries $m_{1}, \cdots, m_{r}$ of $B$ are integers and $\exp z B$ is the diagonal matrix with diagonal entries $\exp z m_{1}, \cdots, \exp z m_{r}$. Since $p(z)=\exp z$, we see that for each $a \in C^{*}, \sigma(\alpha)$ is the diagonal matrix whose diagonal entries are $a^{m_{1}}, \cdots, a^{m_{r}}$.

Let $\rho$ be a holomorphic representation of $G_{A}(L)$ in a complex vector space $U$ and let $\sigma(a)=\rho(0, a)$. Then $\sigma(a)$ is a holomorphic representation of $C^{*}$. We have a basis of $U$ with respect to which every $\sigma(a)$ is represented by a diagonal matrix whose diagonal entries are $a^{m_{1}}, \cdots, a^{m_{r}}$. Denote by $\left\{k_{1}, \cdots, k_{s}\right\}$ the set of distinct integers which appears in $\left\{m_{1}, \cdots, m_{r}\right\}$ and let

$$
U_{i}=\left\{u \in U \mid \sigma(a) u=a^{k_{i}} \cdot u \text { for all } a \in C^{*}\right\}
$$

Then $U=U_{1} \oplus \cdots \oplus U_{r}$. Let $u \in U_{i}$. Then

$$
\sigma(a) \rho(\alpha, 1) u=\rho(0, a) \rho(\alpha, 1) u=\rho(\alpha, 1) \rho(0, a) u
$$

$=a^{k_{i}} \rho(\alpha, 1) u$. Thus we get $\rho(\alpha, 1) u \in U_{i}$. It follows then that each $U_{i}$ is an invariant subspace. Let $\rho_{i}(\alpha, a)$ be the restriction of $\rho(\alpha, a)$ to $U_{i}$. 
Then $\rho_{i}$ is a representation of $G_{A}(L)$ in $U_{i}$ and $\rho_{i}(0, a)=\sigma(a) \mid U_{i}=a^{k_{i}} \cdot 1$. Thus $\rho_{i}$ is homogeneous of order $k_{i}$ and $\rho$ splits into the sum of $\rho_{1}, \cdots$, $\rho_{s}$.

To a holomorphic representation $\rho$ of $G_{A}(L)$ corresponds a holomorphic representation $\rho_{H}$ of $G_{H}(L)$ and a holomorphic vector bundle over $E=$ $V / L$ associated to $\rho_{H}$. In this section we shall denote this vector bundle by $F_{\rho}$ or by $F_{\rho_{A}}$. According to the splitting of the representation $\rho, F_{\rho}$ also decomposes into direct sum. Hence the important case is the case where $\rho$ is an indecomposable representation. By Corollary $9.1 \rho$ is then homogeneous.

THEOREM 9.1. Let $H_{1}$ and $H_{2}$ be Hermitian forms on $V$ such that the imaginary parts $A_{1}$ and $A_{2}$ are rational valued on $L$. Let $\rho_{A_{1}}$ and $\rho_{A_{2}}$ be indecomposable representations of $G_{A_{1}}(L)$ and $G_{A_{2}}(L)$ of order $k_{1}$ and $k_{2}$ respectively. Let

$$
H=k_{1} H_{1}+k_{2} H_{2}, \quad A=k_{1} A_{1}+k_{2} A_{2} .
$$

Then there exists a holomorphic representation $\rho_{A}$ of $G_{A}(L)$ which is homogeneous of order 1 such that

$$
F_{\rho_{A_{1}}} \otimes F_{\rho_{A_{2}}} \cong F_{\rho_{A}}
$$

Proof. Let

$$
\sigma(\alpha)=\rho_{A_{1}}(\alpha, 1) \otimes \rho_{A_{2}}(\alpha, 1)
$$

for all $\alpha \in L$.

Then we have

$$
\sigma(\alpha) \sigma(\beta)=\varepsilon\left\{\frac{1}{2} A(\alpha, \beta)\right\} \sigma(\alpha+\beta)
$$

Put

$$
\rho_{A}(\alpha, a)=\sigma(\alpha) \cdot a
$$

Then $\rho_{A}$ is a holomorphic representation of $G_{A}(L)$ homogeneous of order 1. The theta factor $J$ corresponding to $\rho_{A}$ is given by

$$
J(\alpha, u)=\varepsilon\left\{\frac{1}{2 i} H(u, \alpha)+\frac{1}{4 i} H(\alpha, \alpha)\right\} \rho_{A}(-\alpha, 1)
$$

(see (4.3)). 
We can write this in the form

$$
J(\alpha, u)=J_{1}(\alpha, u) \otimes J_{2}(\alpha, u), \quad(\alpha \in L),
$$

where

$$
J_{j}(\alpha, u)=\varepsilon\left\{\frac{1}{2 i} k_{j} H_{j}(u, \alpha)+\frac{1}{4 i} k_{j} H_{j}(\alpha, \alpha)\right\} \rho_{A_{j}}(-\alpha, 1)
$$

for $j=1,2$. Then $J_{j}$ is the theta factor corresponding to $\rho_{A_{j}}$. This proves that $F_{\rho_{A}} \cong F_{\rho_{A_{1}}} \otimes F_{\rho_{A_{2}}}$.

To prove the next theorem we observe that $L_{1} \times L_{1}^{\prime} \times U_{2 d}$ is a subgroup of $G_{A}(L)$, where $U_{2 d}$ denotes the group of all $2 d$-th roots of unity. For, we have

$$
(\alpha, 0,1) \cdot\left(0, \alpha^{\prime}, 1\right)=\left(\alpha, \alpha^{\prime}, \varepsilon\left\{\frac{1}{2} A\left(\alpha, \alpha^{\prime}\right)\right\}\right) \quad \text { and } \quad d A\left(\alpha, \alpha^{\prime}\right) \in Z .
$$

Moreover the image of this group by the Schrödinger representation $D_{A}$ is a finite group, because we have $D_{A}(\nu, 0,1)=D_{A}(0, \nu, 1)=1$ for $\nu \in N_{1}$, $\nu^{\prime} \in N_{1}^{\prime}$. Therefore we may assume that $D_{A}\left(\alpha, \alpha^{\prime}, 1\right)$ are unitary transformations of $C\left(K_{1}\right)$ (for a suitable inner product) for all $\alpha \in L_{1}, \alpha^{\prime} \in L_{1}^{\prime}$.

THEOREM 9.2. Let $H_{1}$ and $H_{2}$ be Hermitian forms on $V$ such that the imaginary parts $A_{1}$ and $A_{2}$ are rational valued on $L$. Let $\rho_{A_{1}}$ and $\rho_{A_{2}}$ be irreducible holomorphic representations of $G_{A_{1}}(L)$ and $G_{A_{2}}(L)$ respectively and let $k_{1}$ and $k_{2}$ be the orders of homogenuity of $\rho_{A_{1}}$ and $\rho_{A_{2}}$. Let

$$
H=k_{1} H_{1}+k_{2} H_{2}, \quad A=k_{1} A_{1}+k_{2} A_{2} .
$$

Then there exist 1-dimensional representation $\sigma_{1}, \cdots, \sigma_{s}$ of $L$ such that

$$
F_{\rho_{A_{1}}} \otimes F_{\rho_{A_{2}}} \cong\left(L_{\sigma_{1}} \otimes F_{D_{A}}\right) \oplus \cdots \oplus\left(L_{\sigma_{s}} \otimes F_{D_{A}}\right),
$$

where $L_{\sigma_{i}}$ denotes the line bundle associated with $\sigma_{i}$.

Proof. By Theorem 7.1., $F_{\rho_{A_{1}}} \cong L_{\tau_{1}} \otimes F_{D_{k_{1} A_{1}}}$ and $F_{\rho_{A_{2}}} \cong L_{\tau_{2}} \otimes F_{D_{k_{2} A_{2}}}$ and hence $F_{\rho_{A_{1}}} \otimes F_{\rho_{A_{2}}} \cong L_{\tau_{1} \otimes \tau_{2}} \otimes F_{D_{k_{1} A_{1}}} \otimes F_{D_{k_{2} A_{2}}}$. Let

$$
\sigma(\alpha)=D_{k_{1} A_{1}}(\alpha, 1) \otimes D_{k_{2} A_{2}}(\alpha, 1) \quad \text { for all } \alpha \in L
$$

and

$$
\rho_{A}(\alpha, a)=\sigma(\alpha) \cdot a
$$


Then as in the proof of Theorem 9.1, $\rho_{A}$ is a homogeneous representation of $G_{A}(L)$ of order 1 and we have $F_{D_{k_{1} A_{1}}} \otimes F_{D_{k_{2} A_{2}}} \cong F_{\rho_{A}}$. Since $D_{k_{1} A_{1}}(\alpha, 1)$ and $D_{k_{2} A_{2}}(\alpha, 1)$ are unitary for all $\alpha \in L$ as we have seen above, $\sigma(\alpha)$ is also unitary for all $\alpha \in L$. Then the representation $\rho_{A}$ is completely reducible and we have $\rho_{A}=\rho_{1} \oplus \cdots \oplus \rho_{s}$, where each $\rho_{i}$ is irreducible and homogeneous of order 1. Again by Theorem 7.1, we have $F_{\rho_{i}} \cong L_{\lambda_{i}} \otimes F_{D_{A}}$. Then

$$
F_{D_{k_{1} A_{1}}} \otimes F_{D_{k_{2} A_{2}}} \cong\left(L_{\lambda_{1}} \otimes F_{D_{A}}\right) \oplus \cdots \oplus\left(L_{\lambda_{8}} \otimes F_{D_{A}}\right) \text {. }
$$

Letting $\sigma_{i}=\tau_{1} \otimes \tau_{2} \otimes \lambda_{i}$ for $i=1,2, \cdots, s$ we get $F_{\rho_{A_{1}}} \otimes F_{\rho_{A_{2}}} \cong\left(L_{\sigma_{1}} \otimes F_{D_{A}}\right)$ $\oplus \cdots \oplus\left(L_{\sigma_{s}} \otimes F_{D_{A}}\right)$.

Remark. From the proof of Theorem 9.2 we obtain the following rather strange results. Let $m(H)$ be the degree of the Schrödinger representation $D_{H}$ of $G_{H}(L)$. Then $m\left(H_{1}+H_{2}\right)$ divides $m\left(H_{1}\right) \cdot m\left(H_{2}\right)$.

To compute the Chern classes, let $\pi: V \rightarrow E=V / L$ be the covering map. We choose an open covering $\left\{U_{i}\right\}$ of $E$ with the following property: $U_{i}$ are connected and each connected component of $\pi^{-1}\left(U_{i}\right)$ are mapped homeomorphically onto $U_{i}$ by $\pi$. For each $U_{i}$, choose a connected component $\tilde{U}_{i}$ of $\pi^{-1}\left(U_{i}\right)$. Then we have $\pi^{-1}\left(U_{i}\right)=\bigcup_{\alpha \in L} T_{\alpha} \tilde{U}_{i}$, where $T_{\alpha}: V \rightarrow V$ is the translation of $V$ by $\alpha \in L$. Let

$$
\rho_{i}: U_{i} \rightarrow \tilde{U}_{i}
$$

be the inverse of the homeomorphism $\pi: \tilde{U}_{i} \rightarrow U_{i}$.

For each pair $(i, j)$ of indices such that $U_{i} \cap U_{j}$ is non-empty, there exists a unique $\sigma_{j i} \in L$ such that

$$
\rho_{i}(x)=\rho_{j}(x)+\sigma_{j i}
$$

for all $x \in U_{i} \cap U_{j}$.

Let $J$ be a $G L_{r}(C)$-valued theta factor for the torus $E$ and let

$$
g_{i j}(x)=J\left(\sigma_{j i}, \rho_{j}(x)\right)
$$

for all $x \in U_{i} \cap U_{j}$. Then $g_{i j}: U_{i} \cap U_{j} \rightarrow G L_{r}(C)$ is a holomorphic map and $\left\{g_{i j}\right\}$ is a system of transition functions of the vector bundle $F$ over $E$ associated with the factor $J$.

A connection of the vector bundle $F$ is defined by a connection form $\omega=\left\{\omega_{i}\right\}$. Here each $\omega_{i}$ is a $r \times r$ matrix whose entries are 1-forms 
defined on $U_{i}$ and they satisfy the condition

$$
\omega_{j}=g_{i j}^{-1} d g_{i j}+g_{i j}^{-1} \omega_{i} g_{i j} \quad \text { on } \quad U_{i} \cap U_{j} .
$$

We assume that $J$ is of the form

$$
J(\alpha, u)=\varepsilon\left\{\frac{1}{2 i} H(u, \alpha)\right\} C(\alpha),
$$

where $C(\alpha)$ is a constant matrix depending on $\alpha \in L$. Notice that the theta factor associated with a homogeneous representation of $G_{A}(L)$ has this form. Take a basis of $V$ and identify $V$ with $C^{n}$ and write

$$
H(u, \alpha)=\sum_{a, b=1} h_{a b} u_{a} \bar{\alpha}_{b} .
$$

Then $J(\alpha, u)=\exp \left\{\pi \sum_{a, b} h_{a b} u_{a} \bar{\alpha}_{b}\right\} C(\alpha)$ and

$$
g_{i j}(x)=\exp \left\{\pi \sum h_{a b} u_{a}\left(\rho_{j}(x)\right)\left(\overline{\sigma_{j i}}\right)_{b}\right\} C\left(\sigma_{j i}\right) \text {. }
$$

Let

$$
z_{a}^{(i)}=u_{a} \circ \rho_{i}
$$

for each $i$. Then $\left\{z_{1}^{(i)}, \cdots, z_{n}^{(i)}\right\}$ are local coordinates of $E$ on $U_{i}$ and from (9.2) we get

$$
d z_{a}^{(i)}=d z_{a}^{(j)} \quad \text { on } \quad U_{i} \cap U_{j} .
$$

Let $\zeta_{a}$ be the holomorphic 1-form on $E$ such that $\pi^{*} \zeta_{a}=d u_{a}$. Then we have

$$
\zeta_{a}=d z_{a}^{(i)}
$$

on each $U_{i}$.

We get

$$
g_{i j}^{-1} d g_{i j}=\left(\pi \cdot \sum_{a, b} h_{a b}\left(\overline{\sigma_{j i}}\right)_{b} \zeta_{a}\right) \cdot 1_{r}
$$

where $1_{r}$ is the $r \times r$ unit matrix.

Let

$$
\omega_{i}=-\left(\pi \sum_{a, b} h_{a b} \bar{z}_{a}^{(i)} \zeta_{a}\right) \cdot 1_{r}
$$

on each $U_{i}$. Then it is easy to verify that $\omega=\left\{\omega_{i}\right\}$ is a connection 
form. The curvature form $\Omega=\left\{\Omega_{i}\right\}$ is the system of 2-forms such that

$$
\Omega_{i}=d \omega_{i}+\omega_{i} \wedge \omega_{i} \text { on } U_{i}
$$

However we have $\omega_{i} \wedge \omega_{i}=0$ and hence $\Omega_{i}=d \omega_{i}$. Then we get

$$
\Omega_{i}=\left(\pi \sum_{a, b} h_{a b} \zeta_{a} \wedge \bar{\zeta}_{b}\right) \cdot 1_{r} \text { on } U_{i}
$$

and since the left hand side is globally defined we have

$$
\Omega=\left(\pi \sum_{a, b} h_{a b} \zeta_{a} \wedge \bar{\zeta}_{b}\right) \cdot 1_{r}
$$

globally.

The total Chern class $C(F)$ is defined by

$$
C(F)=\operatorname{det}\left(1_{r}+\frac{i}{2 \pi} \Omega\right) .
$$

Since $\Omega$ is of the form (9.6) we get

$$
C(F)=\left(1+\frac{i}{2} \sum_{a, b} h_{a b} \zeta_{a} \wedge \bar{\zeta}_{b}\right)^{r} .
$$

Thus we have

$$
C_{s}(F)=\left(\begin{array}{l}
r \\
s
\end{array}\right)\left(\frac{i}{2} \sum_{a, b} h_{a b} \zeta_{a} \wedge \bar{\zeta}_{b}\right)^{s}
$$

In particular we have

$$
C_{1}(F)=\frac{r i}{2} \sum_{a, b} h_{a b} \zeta_{a} \wedge \bar{\zeta}_{b}
$$

and

$$
C_{s}(F)=\frac{1}{r^{s}}\left(\begin{array}{l}
r \\
s
\end{array}\right) C_{1}^{s}(F)
$$

Now let $F_{\rho_{A}}$ be the vector bundle associated with a holomorphic representation $\rho_{A}$ of $G_{A}(L)$ homogeneous of degree $k$. Then the theta factor is of the form

$$
J(\alpha, u)=\varepsilon\left\{\frac{1}{2 i} k H(\alpha, u)\right\} C(\alpha)
$$

and we get the following theorem. 
THEOREM 9.3. Let $F_{\rho_{A}}$ be the vector bundle over $E=V / L$ associated with a holomorphic representation $\rho_{A}$ of degree $r$ of $G_{A}(L)$ which is homogeneous of order $k$. Let $H$ be the Hermitian form whose imaginary part is $A$ and let

$$
H(u, v)=\sum_{a, b} h_{a b} u_{a} \bar{v}_{b}
$$

for $u, v \in V$. Let $\zeta_{a}$ be the holomorphic 1-form on $E$ whose pullback to $V$ is $d u_{a}(a=1,2, \cdots, n)$. Then we have

$$
C_{1}\left(F_{\rho_{A}}\right)=k r \cdot \frac{i}{2} \sum_{a, b} h_{a b} \zeta_{a} \wedge \bar{\zeta}_{b}, C_{s}\left(F_{\rho_{A}}\right)=\frac{1}{r^{s}}\left(\begin{array}{l}
r \\
s
\end{array}\right) C_{1}^{s}\left(F_{\rho_{A}}\right)
$$

\section{BIBLIOGRAPHY}

[1] J. Igusa, Theta functions, Springer Verlag, 1972.

[2] Y. Matsushima, Fibrés holomorphes sur un tore complexe, Nagoya Math. J. 14 (1959), 1-24.

[ 3 ] H. Morikawa, A note on holomorphic vector bundles over complex tori, Nagoya Math. J. 41 (1971), 101-106.

[ 4 ] D. Mumford, Abelian varieties, Tata Inst. Studies in Math., Oxford Univ. Press, 1970.

[5] T. Oda, (a) Vector bundles on an elliptic curve, Nagoya Math. J. 43 (1971), 41-72, (b) Vector bundles on abelian surfaces, Inventions Math. 13 (1971), 247260.

[ 6 ] A. Weil, Introduction à l'étude des variétés kahleriennes, Paris, Hermann (1958).

[ 7 ] H. Umemura, Some results in the theory of vector bundles, Nagoya Math. J. 52 (1973), 97-128.

Department of Mathematics

University of Notre Dame 